\title{
Towards a Categorical Foundation of Mathematics
}

\author{
M. Makkai* \\ Department of Mathematics \\ McGill University \\ Montreal QC H3A 2K6, Canada \\ makkai@triples.math.mcgill.ca
}

\section{Introduction}

This paper is an expanded version of a talk with the same title given at the Logic Colloquium in Haifa in August, 1995. A year later, at the time of writing these lines, one of the papers, [M2], on which the talk was based has appeared in print. The present paper is, primarily, a somewhat philosophical introduction to the research monograph [M3], the other source of the talk. For understanding the story, it is necessary to keep the contents of [M2] in view; some of [M2] will be recapitulated here. The paper is written for readers with at least a slight familiarity with Category Theory. At certain points, we make remarks referring to more advanced notions; however, the understanding of these is not essential for the main part of the paper.

Some more remarks on the background.

In the abstract for the Haifa talk, another, related development, the material of the papers [M1], was mentioned. In the talk itself, there was no time to enter the subject of sketches. Because of similar considerations of space, sketches will not be discussed in this paper either.

Higher dimensional categories played an important, albeit somewhat hypothetical, role in the talk. They were intended to form the universe of the proposed foundation of the title; on the other hand, their very definition had not yet been given in arbitrary dimensions. In the meantime, a breakthrough has taken place; John Baez and James Dolan proposed (see [BD2]), in a somewhat sketchy form, a definition for the concept of "weak $n$-category", for all natural numbers. I believe that the Baez/Dolan definition is indeed satisfactory. In particular, I think that their "weak $n$-categories" should, collectively for all $n$ and endowed with a suitable attendant structure connecting them to each other, be adopted as forming the universe of the new foundation I am envisaging. Besides, the Baez/Dolan definition also contains very interesting conceptual innovations that make it striking and illuminating even when specialized to the otherwise wellknown case of $n=2$ ! On the other hand, the Baez/Dolan proposal is not the end of the work of even giving the definition of the basic concepts surrounding

\footnotetext{
* The author's research is supported by NSERC Canada and FCAR Quebec
} 
higher dimensional categories, as the authors themselves emphasize in loc.cit. It turns out that the ideas surveyed in the present paper are useful in dealing with the remaining problems concerning higher dimensional categories, and in giving new insights into their structure; I refer to the work [M4] in progress for these matters.

The ideas presented here are related to the so-called structuralist position in the Foundations of Mathematics. P. Benaceraff's paper [Ben1] expresses views that have substantially influenced mine. However, as far as I am aware, the philosophical literature does not contain studies relating Category Theory to the structuralist position, despite some rather obvious points of contact.

From the philosophical point of view, it would be interesting the expand the inquiry, and probe the metaphysics of everyday objects, a multitude of which embody elements of abstraction one is used to in Mathematics. I will refrain from doing so here, but I refer to the paper [M5] (in preparation) for such a discussion.

A final general remark. In this paper, I am interested in the metatheory of an, admittedly incomplete, foundational system. For the purposes of this metatheory, I use all available tools, including ordinary Set Theory.

\section{The universe and the language}

I am going to call the intended new foundation the Structuralist Foundation of Abstract Mathematics, SFAM for short. Before anything else, let me emphasize that SFAM is not completed; later, I'll point to the main missing elements.

I consider SFAM as the result of thinking through, and making explicit, a foundational position behind Abstract Mathematics. I regard Category Theory to be an expression of this position. By Abstract Mathematics, I mean the mathematics of (abstract) structures, such as groups, rings, topological spaces, and in a more advanced vein, e.g., algebraic varieties in Grothendieck's sense.

Category Theory is often seen as a (mere) language for Abstract Mathematics. If we disregard the parenthetical and derogatory "mere", I find that there is much to be said for this view. Indeed, Category Theory and Mathematical Logic are concerned with the language of Mathematics in fundamentally analogous ways.

For one thing, they both have a discernible syntax. In Mathematical Logic, the imperative of providing a totally explicit syntax originates with G. Frege, who established the precise meaning of, and also the possibility of meeting, this imperative. $\mathrm{He}$, and others, developed the languages of various formal axiomatic systems for mathematics. I consider First Order Logic, an all-purpose general language, to be the principal result of these efforts. On the other hand, the overall achievement of Mathematical Logic in clarifying what the qualities of 
the syntax of a satisfactory foundation should be, and in providing a metatheory of syntax, has, I believe, acquired a universal validity, and it cannot be ignored in any foundational inquiry. SFAM is no exception. The conclusion is that the syntax of SFAM should be approachable in an objective way, without having to adopt ontological commitments implicit in SFAM.

Historically, Category Theory has not been concerned with making its syntax explicit to a similar extent. At the basis of Category Theory, we find diagram manipulation as an informal but nevertheless distinct "language". P. Freyd's [F], written out more explicitly in [FS], is an early formalization of a combination of diagram manipulation with ordinary First Order Logic. My work in [M1] aims at pure diagram manipulation, without explicit utilization of connectives or quantifiers. E.g., "doing mathematics inside a topos" is, in my view, the same as applying the formalized diagram manipulation of loc.cit. (via (generalized) 'sketches' as basic syntactical units) to the particular setting of (elementary) toposes (topos theory is, of course, something much more comprehensive; it is the mathematics of toposes). The last fact should be seen as evidence of the power of pure diagram manipulation as a language; note that "doing mathematics inside a topos (with a Natural Numbers Object)" is roughly the same as doing formalized higher order number theory (analysis) (compare [LS]).

First Order Logic with Dependent Sorts (FOLDS), one of the two main themes of this paper, is the result of a search for an "all-purpose" language an applied version of which can be used for SFAM. FOLDS can be seen as a generalization and development of Freyd's [F]; see also [Bl].

Both Mathematical Logic and Category Theory intend to be global approaches to the foundations of mathematics. The Zermelo-Fraenkel formal system of settheory (ZFC), along with its variants, is nowadays almost universally accepted as a global foundation of mathematics; every legitimate piece of mathematics is supposed to be, and as a rule, is, formalizable in ZFC. Category Theory, on the other hand, does not have a closed codification such as ZFC. The globalist character of Category Theory is revealed in its tendency to provide more and more comprehensive notions of "category". The reform through a revision of some concepts, and the extension to achieve a degree of completeness, of the existing framework constitute the other main theme of this paper.

The standard ZFC foundation possesses two prominent features: on the one hand, the intuitive appeal and clarity of its universe of discourse, the universe of (von-Neumann) pure (or regular) sets, and on the other hand, the transparency and simplicity of its language, (applied) first order logic. While Category Theory is governed by compelling concepts and intuitions, sometimes sharply at variance with the standard foundation, it does not provide clear-cut answers to the questions "what is the universe and what is the language of abstract mathematics?" The present paper tries to give partial answers to those questions in the spirit of Category Theory. 


\section{Abstract sets}

The divergence between the standard foundation and SFAM begins with their views on sets. In SFAM's view, the world of mathematics is big and heterogeneous; a set is a relatively orderly part of the world in which an equality predicate reigns. The elements of a set are individuated with respect to each other. However, there is no global equality present for all things simultaneously. An equality predicate is an equivalence relation on the given set. In fact, the set is the underlying collection together with its equality predicate.

This fact is part of the explanation of why the adjective "structuralist" appears in the name of SFAM; already sets are a certain kind of structure, and other concepts of totality will be increasingly complex structures. I take it to be a tenet of structuralism that everything accessible to rational inquiry is a structure; the conceptual world consists of structures.

Another fact about sets concerns the membership predicate. In fact, in SFAM, it is not a full-fledged predicate. It is not meaningful, that is, not grammatical, to write $x \in a$ for a set $a$, and an arbitrary variable $x$. In other words, it is not meaningful to ask, for a set $a$ and an arbitrary thing $x$, whether or not $x$ is an element of $a$. Rather, a set $a$ is a type, and a variable $x$ may be declared to be of type $a$. " $x \in a$ " will not be a predicate subject to free manipulation with the connectives and quantifiers as full-fledged predicates will be; for instance, we will never write $\neg(x \in a)$. The statement " $x \in a$ " will have the role of defining contexts of variables, in the style of P. Martin-Löf [M-L] and Cartmell [C]. Thus, SFAM is a type theory; the difference to ordinary (unramified, simple, Russell-Ramsay) type-theory is that types are variable; more precisely, they (may) depend on variables.

Thus in SFAM, both equality and membership are denied the free reign they enjoyed in the standard foundation.

In SFAM, we will adhere to a very strict idea of "type". An entity belonging to a type cannot be discussed without reference to the type; the type logically precedes the entity, and the type is inseparable from the entity.

As we said, a set is a type; actually, for a set $a$, we have the type $\mathrm{E}(a)$ of its elements. The type $\mathrm{E}(a)$ a dependent type, has the invariable element $\mathrm{E}$, and the variable element $a$ in it. When a variable $x$ is declared to be of type $\mathrm{E}(a)$, in symbols $x: \mathrm{E}(a)$, which is synonymous with $x \in a$, the relationship established between $x$ and $a$ is completely rigid, or invariable. For instance, it will not be possible for a variable to be of two types that are different syntactically. (This is in contrast to [M-L] and [C]; see also below). What we just said is the syntactical counterpart of the metaphysical intuition that an element of a set may enter into relationships with other things only as an element of the set, only through the set itself. 
The above example $\mathrm{E}(a)$ of a dependent type is only one of an infinite number of such types in SFAM.

Dependent types are well-known from P. Martin-Löf's work [M-L]. See also J. Cartmell [C] that pertains, similarly to SFAM, to an essentially first-order framework, albeit without quantifiers. However, both the philosophy and the actual syntax of dependent types I am proposing to use for SFAM differ from those in the cited sources. I have not been able to find in the literature views on types that accord in all the essentials with the views I am advocating here.

Note that the character of a set as "type" in the way described above is, at first sight, at variance with the idea behind the comprehension principle of Set Theory. In that principle, we have, in the first place, certain entities, of a certain arbitrary type, say, and in the second place, we form a set whose elements are the given entities. This would mean, in our context, attributing a new type, namely $\mathrm{E}(a)$ for the set $a$ thus "comprehended", to entities of an already established type. This, directly at least, is not allowed in SFAM. Instead, we will have a somewhat elaborate "relational structure" connecting the entities of type $\mathrm{E}(a)$ with the original entities. This structure will also reflect our specific way of dealing with equality.

As a result, comprehension, although possible, is much less "cheap" in SFAM than in Set Theory. This is not such a bad thing, considering the treacherous nature of (unrestricted) comprehension, trivial-looking instances of which lead to contradictions (Russell's paradox). While axiomatic Set Theory is based on one selection of instances of (unrestricted) comprehension, ones that are "Cantorian", or fit the "limitation of size" doctrine (see [H]), SFAM will be based on another selection of instances, including ones that (or rather, whose direct counterparts) are not admissible in Set Theory. SFAM is envisaged to be a "nonCantorian" theory of totalities. The actual laying down of a reasonably complete set of axioms of SFAM awaits future work.

In Abstract Mathematics, we find the intuitive idea of abstract set, one whose elements are characterless, nevertheless distinct, points. An "abstract" structure is one whose underlying set is an abstract set. An "abstract" group is given by an abstract set $A$, and by a binary operation on $A$, assumed to satisfy certain conditions, the group axioms. The elements of the group, which are mere points to begin with, enter into meaningful relations via the equality predicate of the underlying set and the group-operation, which is a function of the form $A \times A \rightarrow A$.

The sets of SFAM are abstract sets in the above intuitive sense. But then, one might object that in Mathematics, even in Abstract Mathematics, we also need "non-abstract" structures, ones whose underlying sets are not abstract sets. For instance, we want to consider the group of automorphisms of a given structure; the elements of this group are certainly not characterless points. 
The answer to this is that in the group of automorphisms, the elements are characterless surrogates of the automorphisms, rather than the automorphisms themselves. There is an additional structure ensuring the presence of a relation which establishes a bijection between the automorphisms and the characterless points. It is as if we had abstracted away from the concrete nature of the automorphisms to get the elements of the group.

The reader who is familiar with mathematics done in a topos will recognize that, in fact, in a topos all objects behave like abstract sets. For instance, the categorical product of two objects, which corresponds to the idea of the Cartesian product of two sets, is just another object, equipped with the two projection maps; the complex of the three objects and two arrows involved have a so-called universal property characteristic of products (see [ML]). In the "topos of abstract sets", the product-set, instead of having the ordered pairs $(a, b)$ with $a$ and $b$ elements of the two sets we began with, has elements that are characterless points; however, the projection maps together with the universal property characteristic of products ensure that those elements are in fact in a "structural", built-in bijection with the said ordered pairs. The way the topos structure deals with specifying the powerset of an abstract set is similar; the powerset is just another abstract set, but there are "structure maps" around ensuring that its elements are in a bijection with the subsets of the given set. Note that the universal properties alluded to are statements formulated by the use of quantifiers in the first-order language of the category; they express a relation of the arrows involved in the product or in the powerset to the totality of all arrows in the category. Thus the "internal" structure of the Cartesian product given by the fact that its elements are suitable ordered pairs is replaced by an "external" relation of the product set and the projections to other sets and functions; a similar thing can be said concerning the powerset.

In a topos, it is possible to construct the group of automorphisms of a structure (e.g., field) in the topos. This will be an abstract group; however, the construction will provide an accompanying structure of various maps giving a faithful representation of the fact that the elements are in a bijective relation with the automorphisms of the structure, and that under this relation, the group-operation corresponds to composition of automorphisms.

On the other hand, the regular (well-founded) sets of ZFC set-theory are concrete sets. A regular set has elements that are themselves are regular sets. A regular set is individuated by its elements, rather than the other way around as with abstract sets. Each element $x$ of a regular set $A$ is individuated 'completely' by the membership relation, and by the equality relation deduced from it, without reference to $A$. The well-founded nature of the membership relation ensures that the determination of $x$ is indeed independent of $A$. Each regular set $x$ can be uniquely picked out from the totality of all regular sets by structural properties alone, indeed by referring only to elements of $x$, to elements of the elements of $x$, and so on, through the transitive closure of $x$. In particular, the structured 
totality of regular sets has no non-trivial automorphism. In a way, regular sets are at an opposite end from being "abstract"; they are "concrete".

Here is an illustration how Abstract Mathematics gets into conflict with "concrete sets". Consider a hypothetical book on group theory, for instance, and suppose we had something like the following in it. "Let $G$ and $H$ be arbitrary groups, and consider the intersection $X$ of their underlying sets", after which a construction involving the data mentioned, in particular the set $X$, is described. I am sure everyone used to group-theory should be taken aback by such a passage. One would say that what the common elements of the groups are is purely incidental to the essence of the groups, and thus the datum of the intersection $X$ can have no significant role in a mathematically meaningful construction based on the given groups $G$ and $H$. Now, the intersection of two sets is a typical operation on sets that is meaningful only under the free reign of an (untyped) membership relation, but not for abstract sets. In a more advanced manner, we would say that the proposed construction is very likely not going to be invariant under isomorphism of groups; we take up this point later.

When one is told, as in Mathematics explicitly based on Set Theory one is, that "the underlying sets of structures are regular sets", one may acquiesce in that view on the basis that it is necessary (one is told) for having a consistent foundation. But one will feel that the original intuitions of Abstract Mathematics have been altered.

\section{Functions, categories and isomorphisms.}

The usual kind of equality of sets is not meaningful in SFAM. That equality, defined through extensionality, relies on the untyped (free-reigning) membership relation, or alternatively, on the untyped equality relation of all things, neither of which is available in SFAM. (We may put the "definition" of equality of sets in two ways:

$$
\begin{gathered}
X=Y \Leftrightarrow \forall x(x \in X \leftrightarrow x \in Y) \\
X=Y \Leftrightarrow \forall x \in X \cdot \exists y \in Y \cdot y=x \quad \& \quad \forall y \in Y \cdot \exists x \in X \cdot x=y .
\end{gathered}
$$

(In the first form, the untyped $\epsilon$, in the second, the untyped = appear.)

The upshot is that we do not have available a set of sets; sets do not form a set (not, in this instance, for size considerations, but rather, because of the lack of an available equality of sets). Rather, sets will form a new type of totality: a category.

What connects sets is functions. Functions are a new kind of entity; for any sets $X$ and $Y$, we have the idea of a function from $X$ to $Y$. We write $f: X \rightarrow Y$ for " $f$ is a function from $X$ to $Y$ ", i.e., " $f$ is a function with domain equal to $X$, and range included in $Y$ ". "Function" is a primitive concept in SFAM, 
unlike "function" in the standard foundation. A further primitive is composition of (composable) functions, making sets and functions into a category in the usual sense. Below, in section 8 , we will see the exact type-theoretic treatment of the concept of category that is adopted in SFAM.

(I do not see any a priori justification why we must have, e.g., functions as primitives. For instance, we could take instead relations, a generalization of function. However, when doing so we end up with essentially the same theory. The Category of Sets as sets-and-functions and the "Category" of Sets as setsand-relations are interdefinable. On the other hand, there are a posteriori justifications for preferring functions over relations; the theory is nicer with functions than with relations. We have to consider it a genuine discovery that the "right" structure of sets is the category of sets. This discovery is due to F. W. Lawvere; it culminates in the concept of the Lawvere-Tierney concept of (elementary) topos $[\mathrm{J}],[\mathrm{MM}]$.

Set, the category of sets (and functions) gives rise to many further categories, such as the category of groups, that of topological spaces, etc. In the General Algebra of structures (("universal") algebras, relational structures, topological structures, etc.), the categories of those structures are nowadays recognized to play a basic, albeit possibly "trivial" role, the role of a "mere language". In [M6], I tried to show that the exposition of an algebraic version of propositional logic, involving as it does Boolean algebras, Heyting algebras, etc., is considerably enlightened by the systematic use of the categories of the various kinds of structure involved. In general, I subscribe to the view that General Algebra requires Category Theory.

In any category, we have the notion of isomorphism. An isomorphism of objects $X$ and $Y$ is a pair of arrows $X \underset{g}{\stackrel{f}{\rightleftarrows}} Y$ such that $g f=1_{X}$ and $f g=1_{Y}$ (Note that we have equality of arrows here. Let us note that equality will be allowed only for arrows with the same domain and the same codomain. For more details on the SFAM notion of "category", see section 8).

A single arrow $X \stackrel{f}{\longrightarrow} Y$ is called an isomorphism (or if necessary, an isomorphism arrow) if there is a (necessarily unique) $g$ making the pair $(f, g)$ an isomorphism. We say that $X$ and $Y$ are isomorphic if there is an isomorphism between them. Note that the isomorphism arrows in a given category form another category, called the underlying groupoid of the given category (in a groupoid, every arrow is an isomorphism). The usual concepts of isomorphism for various mathematical structures ("homeomorphism" for topological spaces, for instance) are the same as isomorphism in the respective categories.

One of the first intuitions of Abstract Mathematics is that two isomorphic structures are, essentially, the same. SFAM adopts isomorphism of objects in a category (in particular, isomorphism, that is, equipollence, of sets) to play the role of equality for those objects, in the sense that 
(Principle of Isomorphism) all grammatically correct properties of objects of a fixed category are to be invariant under isomorphism.

Here, a property $P(Y)$ of the variable object $Y$ in a given category is invariant under isomorphism if $P(Y)$ and $Z \cong Y$ imply $P(Z)$.

The just-stated principle is the analog, formulated for isomorphism in place of ordinary equality, of Leibniz's indiscernibility of identicals, the usual axiom of equality that allows to substitute any thing by any other thing equal to it. At this point, when I am not presenting a closed codification of SFAM, the Principle of Isomorphism appears as a requirement on the language of SFAM. Of course, the principle restricts the possible expressions allowed in SFAM. In particular, "ordinary" equality of objects (in particular, "ordinary" equality of sets) is immediately disallowed in SFAM, since for a fixed object $X$, the predicate $Y=X$ of the variable $Y$ is, obviously, (usually) not invariant under isomorphism.

The last-stated consequence of the Principle of Isomorphism is an expression of a wide-spread understanding in Category Theory that excludes equality of objects in a category from meaningful concept-formation. But more positively, the Principle of Isomorphism itself appears to be a generally accepted idea in Abstract Mathematics. We say things like these:

"two groups that are isomorphic share all structural properties; they are structurally indistinguishable";

"(abstract) group theory studies only structural properties of groups, ones that are invariant under isomorphism".

The basic character of the Principle of Isomorphism is that of a constraint on the language of Abstract Mathematics; a welcome one, since it provides for the separation of sense from nonsense. But the fact that isomorphism is the real equality in Abstract Mathematics is also an experience. E.g., the structure theorems of the subject essentially always assert the isomorphism, often by "canonically given" isomorphism mappings, of a "source" structure to a "target" structure, the latter possessing a specific description; one almost never finds the equality in the sense of underlying set theory of those two structures asserted.

The aim of SFAM is to have a self-contained language in which only structural properties of, say, groups can be formulated.

Let us note that in the practice of Category Theory, we usually expect that all meaningful properties of an object in a fixed category are invariant under isomorphism.

Some more remarks on isomorphism as equality. Unlike ordinary equality, isomorphism as a relation between objects is not a primitive notion; it is derived, 
by logical means, from the primitives of the concept of category. Indeed, we may say that the individuation of objects does not happen through the relation of isomorphism, but rather, by the total structure of isomorphism arrows, the underlying groupoid of the category. Because of this, the Principle of Isomorphism as stated above is the tip of an iceberg; it does not describe fully what is going on.

As it happens, in category-theoretic concept-formation (such as "monoidal category", for instance), it is never the relation of isomorphism (of the objects $A \otimes(B \otimes C)$ and $(A \otimes B) \otimes C$, for instance) that appears directly; rather, we pick out "canonical" isomorphism arrows (for instance, the associativity isomorphisms $\left.\alpha_{A, B, C}: A \otimes(B \otimes C) \stackrel{\cong}{\longrightarrow}(A \otimes B) \otimes C\right)$ that we incorporate into the concept as part of the data.

As a further example, consider the fact that the construction of the direct product $G \times H$ of groups is invariant under isomorphism. This, in actual fact, says more than what the above formulation of the Principle of Isomorphism states; it is more than just saying that if $G \cong G^{\prime}$ and $H \cong H^{\prime}$, then $G \times H \cong G^{\prime} \times H^{\prime}$. It also contains the fact that any pair of automorphisms $g: G \stackrel{\cong}{\longrightarrow} G, h: H \stackrel{\cong}{\longrightarrow} H$ induces an automorphism $g \times h: G \times H \stackrel{\cong}{\longrightarrow} G \times H$.

The structure of individuation of objects in a category (in particular, that of sets) is something more elaborate than a mere primitive relation.

Probably this is a good place to remark that Abstract Mathematics is not so much a restricted part of Mathematics, the one dealing with abstract structures, as a specific way of looking at all that is mathematical. The natural numbers are indispensable in any mathematics, abstract or concrete. Now, the view of SFAM of the natural numbers is that they are the elements of any structure of the form $(N, 0 \in N, S: N \rightarrow N)$ that is an initial object in the category of all structures of the said form (this is what is called the (Lawvere) Natural Numbers Object; it is the same, under a certain amount of set theory, as a model of the (categorical) second order Peano Axioms). Any object isomorphic to an initial object is again one; the concept of Natural Numbers Object is invariant under isomorphism. In SFAM, certainly there is no uniquely determined totality of all natural numbers; and the names "one", "two", ... do not name absolutely fixed entities. P. Benaceraff of [Ben1] should approve of SFAM's dealing with the natural numbers, although Benaceraff of [Ben2] may not.

\section{A revision of category theory}

We are facing the question of the totality of all categories. What kind of totality is this? It cannot be a category itself. The argument for this is similar to the one we saw above concerning why sets do not form a set. More specifically, the concept of an isomorphism between two categories is clearly seen to involve the idea 
of equality of objects; how would one otherwise formulate the 1-1 character of the isomorphism? Therefore, categories cannot be isomorphic; as a consequence, they cannot be objects of a category.

As we expect from the progression from the sets as totalities with equality to categories, the kind of totality categories form will be a more complicated version of category; in fact, it will be a "2-dimensional category" of a certain kind. But before we get to the general notion of "2-dimensional category", we need the particular ingredients of the "2-dimensional category" of categories. And now we find a surprise.

The classical concept of morphism of categories is functor. Categories, functors, together with natural transformations, endowed with several composition operations, form the classical 2-category Cat of categories (at the moment, we are ignoring size considerations); see [ML]. However, upon inspection, the concept of "functor" turns out to be deficient from the point of view of SFAM.

A functor, as a function assigning (among others) a definite value-object to an argument-object, is involved with equality of objects, via the requirement that the value-object be uniquely determined. In a more positive manner, we should have, according to the Principle of Isomorphism stated above, that

$\left.{ }^{*}\right)$ for a "functor" $F: \mathbf{X} \rightarrow \mathbf{A}$, and an object $X \in \mathbf{X}$, if $A$ is a value of $F$ at $X$, and $B$ is another object of $\mathbf{A}$ isomorphic to $A, B \cong A$, then $B$ too is a value of $F$ at $X$.

Of course, functors will not satisfy this requirement. The notions of "anafunctor", and "saturated anafunctor", are introduced to deal with the problem.

Here are some definitions, made in ordinary Set Theory, for the sake of definiteness. Let $\mathbf{X}, \mathbf{A}$ be categories. An anafunctor $F: \mathbf{X} \stackrel{a}{\longrightarrow} \mathbf{A}$ is given by data as in (i) and (ii):

(i) a set $|F|(X, A)$ for any $X \in O b(\mathbf{X}), A \in O b(\mathbf{A})$

(the elements of $|F|(X, A)$ are called specifications; $s \in|F|(X, A)$ specifies $A$ as a possible value of $F$ at $X$ );

(ii) for $(f: X \rightarrow Y) \in \operatorname{Arr}(\mathbf{X}), A, B \in O b(\mathbf{A}), s \in|F|(X, A), t \in|F|(Y, B)$, an arrow $F_{s, t}(f): A \rightarrow B$.

The data are required to satisfy conditions (iii) to (v):

(iii) for any $X \in O b(\mathbf{X})$, there is $A \in O b(\mathbf{A})$ such that $|F|(X, A)$ is inhabited (non-empty); 
(iv) $s \in|F|(X, A) \Rightarrow F_{s, s}\left(1_{X}\right)=1_{A}$;

(v)

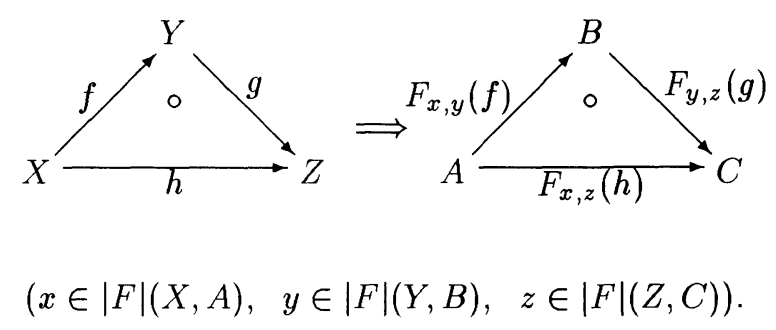

Although at the time of working on [M2] I did not know about the fact, G. M. Kelly $[\mathrm{K}]$ had already defined the notion that I call anafunctor, in a brief passage, as a side remark. He did not name it, but he characterized the concept as a description of a common situation in which one, seemingly possessing all the data needed, wants to define a functor, but in which, for the specification of the functor, one still needs to make arbitrary choices that usually require the axiom of choice. What I do in [M2], and Kelly did not do, is develop the theory of anafunctors, and thereby demonstrate that one does not have to make those arbitrary choices, and that one can live with anafunctors in place of functors. The result is a category theory which is more "canonical" than the the usual one. In technical terms, when using anafunctors, to a large extent one can avoid the use, ubiquitous in the usual theory, of the Axiom of Choice.

Let me emphasize that [M2] is developed in ordinary Gödel-Bernays set-theory, mostly without the Axiom of Choice, but at any rate, with a careful keeping track of the uses of choice, and even of the principle of the excluded middle (which is regarded, after R. Diaconescu [D], a particular case of Choice). In SFAM proper, the problem with functors is bound to be graver, and the need for something like anafunctors greater, since the kind of choice we need, Global Choice, cannot even be meaningfully stated in SFAM. The reason is precisely the lack of equality on the entities, objects in a category, among which we want to make unique choices. In contrast, the local, (set-)version of choice remains perfectly meaningful in SFAM.

Whereas the anafunctors are more general than functors, saturated anafunctors, the ones we really want because of the requirement $\left({ }^{*}\right)$, are a special class within anafunctors, almost disjoint from that of functors; I am not aware in the literature of a mention of the property of saturation, under any name.

To introduce this concept, let $F: \mathbf{X} \stackrel{a}{\longrightarrow} \mathbf{A}, X \in O b(\mathbf{X}), A \in O b(\mathbf{A})$ and $s \in|F|(X, A), t \in|F|(X, B)$. Then we have $f=F_{s, t}\left(1_{X}\right): A \rightarrow B$, and $g=F_{t, s}\left(1_{X}\right): B \rightarrow A$, and the laws easily imply that $f$ and $g$ are inverses of each other; $(f, g)$ is an isomorphism. Thus, we see that any two possible values of $F$ at an object $X, A$ and $B$ here, are isomorphic. Now, forget $t$ but keep the 
other data; and take an arbitrary isomorphism $f: A \stackrel{\cong}{\longrightarrow} B . F$ is saturated if there is a unique $t \in|F|(X, B)$ such that $f=F_{s, t}\left(1_{X}\right)$, for all choices of the parameters $X, A, B$ and $s$ involved.

I regard this condition as the correct rendering of the Principle of Isomorphism, for the notion of (ana)functor. The principle expresses that if we have one possible value of $F(X)$, and we change it by a specific isomorphism to another object, then the new object is also a possible value of $F(X)$, by a uniquely determined specification. We see again that the Principle of Isomorphism in its general form by itself is only the tip of an iceberg. Simply requiring of an anafunctor that for a fixed argument-object, being a possible value-object be invariant under isomorphism, although logically possible, would clearly not give a good notion.

Let me mention that, as a consequence of the definition, we can see that in a saturated anafunctor one can also change the argument-object along an isomorphism, with keeping the value-object fixed.

To illustrate the effect of anafunctors, consider a category $C$ with binary products. In category theory, we want to consider the product functor

$$
\begin{gathered}
P: \mathbf{C} \times \mathbf{C} \rightarrow \mathbf{C} \\
(X, Y) \mapsto X \times Y
\end{gathered}
$$

Of course, we do not have, in general, a well-defined product $X \times Y$; we only have the notion of product diagram, given by a universal (extremal) property: certain diagrams of the shape

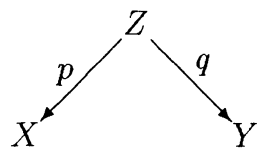

qualify as products, and there is at least one such for any $X$ and $Y$. This is the point where one uses "global choice", and one picks a particular product-object $Z$, and projections $p$ and $q$, and call them $X \times Y, \pi_{X, Y}$ and $\pi_{X, Y}^{\prime}$, respectively. Having made these choices, the definition of $P$ is natural. It is also true that it does not matter how we make the choices, since the resulting functors are all isomorphic (by natural transformations). The fact remains, however, that we need Choice to define $P$. On the other hand, we have the anafunctor, also called $P$, defined as follows: $P: \mathbf{C} \times \mathbf{C} \stackrel{a}{\longrightarrow} \mathbf{C}:$

$$
|P|((X, Y), Z)=\{(p, q):(1) \text { is a product }\}
$$

for $(p, q) \in|P|((X, Y), Z), \quad(r, s) \in|P|((A, B), C), \quad f: X \rightarrow A, \quad g: Y \rightarrow B$ : $P_{(p, q),(r, s)}((f, g))=$ the unique $h$ for which $P$ is in fact a saturated anafunctor. 


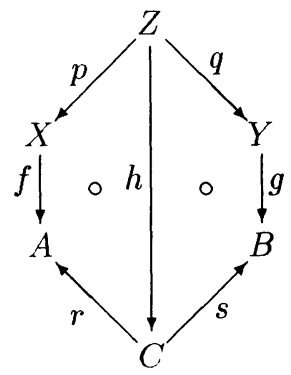

One defines natural transformations of anafunctors. For fixed categories $\mathbf{X}$ and $\mathbf{A}$, anafunctors $\mathbf{X} \stackrel{a}{\longrightarrow} \mathbf{A}$ and their natural transformations form a category Ana $(\mathbf{X}, \mathbf{A})$. Every $F: \mathbf{X} \stackrel{a}{\longrightarrow} \mathbf{A}$ is canonically isomorphic in $A n a(\mathbf{X}, \mathbf{A})$ to a saturated anafunctor $F^{\#}$; saturated anafunctors are enough. Every ordinary functor is an anafunctor in an obvious way; and by using Choice, but not without it, every anafunctor is isomorphic to a functor. (This is a way of putting what Kelly was after; note however that for stating this we need the notion of natural isomorphism of anafunctors, which Kelly did not consider.) In section 10 , we will define $F^{\#}$ for an ordinary functor $F$.

In [M2], "basic category theory" is redone with using (saturated) anafunctors in place of functors. For instance, one obtains a bicategory (see the next section) SanaCat of categories, saturated anafunctors, and their natural transformations; under the Global Axiom of Choice, SanaCat is biequivalent to Cat, the classical 2 -category of categories.

\section{2-dimensional categories}

Abstracting from categories, functors and natural transformations, together with the basic operations on them, leads to the concept of 2-category; see [ML]. In a 2 -category $\mathcal{A}$, we have three kinds of entities: objects (0-cells) $\mathbf{A}, \mathbf{B}, \cdots$ arrows (1-cells)

$$
F: \mathbf{A} \rightarrow \mathbf{B}
$$

(thus, each arrow has a source (domain), and a target (codomain)), and 2-cells as in

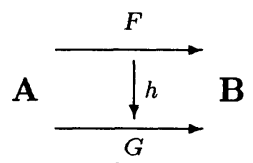

(thus, a 2-cell has parallel source and target 1-cells). For a fixed pair of 0-cells $\mathbf{A}, \mathbf{B}$, the 1-cells (1), and the 2-cells (2) form a category $\mathcal{A}(\mathbf{A}, \mathbf{B})$; this involves the so-called vertical composition of 2-cells:

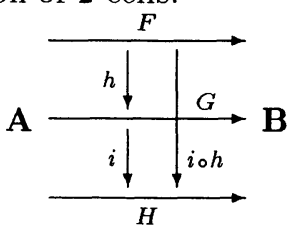


Furthermore, for any 0-cells $\mathbf{A}, \mathbf{B}, \mathbf{C}$, we have a composition functor

$$
\begin{aligned}
& { }^{\circ} \mathbf{A}, \mathbf{B}, \mathbf{C}: \mathcal{A}(\mathbf{A}, \mathbf{B}) \times \mathcal{A}(\mathbf{B}, \mathbf{C}) \rightarrow \mathcal{A}(\mathbf{A}, \mathbf{C}) \\
& (\mathbf{A} \stackrel{F}{\longrightarrow} \mathbf{B}, \mathbf{B} \stackrel{G}{\longrightarrow} \mathbf{C}) \mapsto \mathbf{A} \stackrel{G F}{\longrightarrow} \mathbf{C}
\end{aligned}
$$

involving the so-called horizontal composition of 2-cells:

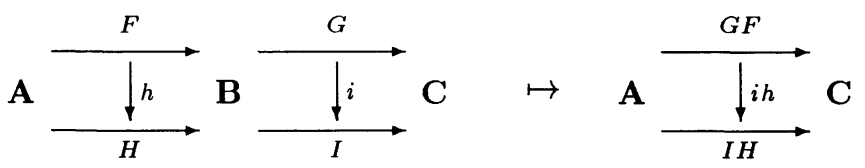

We require that composition be associative; in particular, in the situation

$$
\mathbf{A} \stackrel{F}{\longrightarrow} \mathbf{B} \stackrel{G}{\longrightarrow} \mathbf{C} \stackrel{H}{\longrightarrow} \mathbf{D},
$$

we have

$$
H(G F)=(H G) F .
$$

(To complete the definition of "2-category", certain stipulations on identity arrows need to be added only.)

In [Be], the concept of bicategory is introduced. As Benabou demonstrated, bicategories occur naturally at many points of Category Theory; in particular, the concept of (not necessarily strict) monoidal (or tensor) category (see [ML]) is the same as a one-object bicategory. The concept of bicategory can be seen as the result of replacing equalities of objects in a category by canonical (structural) isomorphisms, a move in the direction of SFAM. In particular, one way a bicategory differs from a 2-category is that, in place of the required equality (3), in which $H(G F)$ and $(H G) F$ are objects of the category $\mathcal{A}(\mathbf{A}, \mathbf{D})$, we take up, as part of the structure, an isomorphism 2-cell

$$
\alpha_{F, G, H}: H(G F) \stackrel{\cong}{\longrightarrow}(H G) F,
$$

an isomorphism in the category $\mathcal{A}(\mathbf{A}, \mathbf{D})$. The complete definition of "bicategory" requires further canonical isomorphisms, and also, the so-called coherence conditions that connect, e.g., various instances of $\alpha_{-,-,-}$.

As it was mentioned at the end of the last section, when, working in ordinary Set Theory, we replace functors by (saturated) anafunctors, and make the necessary other adjustments, we obtain from Cat a bicategory AnaCat (SanaCat). In particular, now we do not get a 2-category.

Just like Set spawns a large class of other categories of set-based structures, so does $C$ at give rise to 2-categories, and bicategories, of structured categories. In $[\mathrm{S}]$, we find the view that, similarly to the need for the theory of categories in General Algebra that treats of set-based structures, the algebra of structured categories should be based on the theory of bicategories. In the work of the 
Australian School (G. M. Kelly, R. Street, and others), we find a realization of this idea.

In any bicategory, in particular, in any 2-category, there is a notion of equivalence (of 0-cells), a higher-dimensional analog of isomorphism in a category. For 0-cells $\mathbf{A}, \mathbf{B}$, an equivalence of $\mathbf{A}$ and $\mathbf{B}$ is a quadruple $(F, G, i, j)$ as in

$$
\mathbf{A} \underset{G}{\stackrel{F}{\rightleftarrows}} \mathbf{B} \quad i: G F \stackrel{\cong}{\longrightarrow} 1_{\mathbf{A}}, \quad j: F G \stackrel{\cong}{\longrightarrow} 1_{\mathbf{B}}
$$

involving the identity 1 -cells $1_{\mathbf{A}}: \mathbf{A} \rightarrow \mathbf{A}, 1_{\mathbf{B}}: \mathbf{B} \rightarrow \mathbf{B}$. We write $(F, G, i, j):$ $\mathbf{A} \simeq \mathbf{B}$. A 1-cell $F$ is called an equivalence (arrow) if it can be extended to an equivalence $(F, G, i, j)$. Similarly for the pair $(F, G)$ being an equivalence. A and $\mathbf{B}$ are equivalent, in symbols $\mathbf{A} \simeq \mathbf{B}$, if there is an equivalence of them.

The usual concept of equivalence of categories is that of equivalence in Cat. It is generally recognized, in exact analogy to sets and set-based structures in relation to the notion of isomorphism, that the "right notion" of "equality" for categories, resp. category-based structures is equivalence of categories, resp. equivalence in the corresponding bicategory. This principle acts, again, in two different ways. First, as the constraint on properties of objects in a bicategory, which we may call the Principle of Equivalence, asserting that

\section{any (meaningful) property of an object in a bicategory is invariant under equivalence.}

Secondly, as the experience that usually, especially in "serious" representation theorems, one gets that a given category can be represented in a certain desired way up to equivalence, but not up to isomorphism.

It is obvious that for the purposes of SFAM, the concept of bicategory requires a (further) modification: the composition functors (3) have to be replaced by (saturated) anafunctors, and a similar, more minor change, is needed with respect to the identity 1-cells. The resulting (saturated) anabicategories are worked out in [M2].

By making certain "arbitrary" choices, any anabicategory can be reduced to a bicategory; and vice versa, there is a canonical process of saturating any anabicategory, in particular, any bicategory. Thus, in the presence of the Global Axiom of Choice, saturated anabicategories are essentially "equivalent" (in fact, also in a technical sense, (bi)equivalent) to bicategories.

It is instructive to see the definition of the monoidal category, that is, one-object bicategory, of $R$-modules, for $R$ any commutative ring, and compare it with its saturated anaversion. The latter can be defined directly, by appropriately modifying the usual definition. In fact, the modifications are seen to consist in eliminating all arbitrary choices the usual definition has to make (see [ML]). 


\section{Higher dimensional categories}

The foregoing strongly suggests that there should be an infinite hierarchy of " $n$ dimensional categories", for $n=0,1,2, \cdots$, such that $n$-dimensional categories "form" (are the objects of) an n+1-dimensional category. We have discussed this hierarchy for $n=0$ ('set'), 1 ('category') and 2, in which case several concepts have been advanced, with the final suggestion that from the point of view of SFAM, 'saturated anabicategory' should be the right one.

Let me emphasize that from the point of view of SFAM, the said hierarchy is one of ever increasing generality. For example, every set is, or gives rise to, a category, namely the discrete category with objects the elements of the set, and only identity arrows. On the other hand, a category does not give rise to a set; the totality of objects is not a set; it is not provided, by the data of the category, with an equality predicate. More strongly, from the point of view of SFAM, a category is not an ordinary, "set-based" structure. This can be seen from the fact that the structural identity relation for categories is not isomorphism, but something else, namely equivalence of categories.

The notion of $n$-category, the direct analog of 2-category for arbitrary $n$, is rather clear. In fact, we have the general theory of enriched categories [K2]; and, recursively, an $n+1$-category is a category enriched in the (ordinary) category of $n$-categories.

The case of the hierarchy continuing the concept of bicategory, which, following [BD1], we will call weak $n$-category, is quite a different story; in fact, no definition of "weak $n$-category" has gained general acceptance yet (however, see below for some good news in this regard). The general idea of what a weak $n$-category should be is reasonably clear: one should "weaken" the definition of $n$-category, by replacing all equalities by "canonical" arrows: arrows that are part of the structure. The difficulty is in giving explicitly the so-called "coherence conditions" the canonical arrows should satisfy. In [BD1] we read: "It is clear that new ideas are needed to do so without a combinatorial explosion, since already the explicit definition of a tricategory [in [GPS]] takes six pages, and that of a triequivalence 13 pages!".

Let us note that the present high interest in weak $n$-categories, and in higher dimensional category theory in general, is driven mainly by actual and potential applications in topology, theoretical physics, and in particular, quantum groups; it has relatively little to do with the foundational concerns of the present paper. See [BD1].

Upon contemplation, one sees, already for $n=2$ that the "real" definition of bicategory is not the official one, but rather one that stipulates "all coherence conditions" at once, i.e. (roughly) that "all diagrams" built, in the "free" or 
"generic" case, from instances of the associativity isomorphisms and other canonical isomorphisms should commute. It is an important coherence theorem (due to $\mathrm{S}$. Mac Lane, formulated for the one-object case, that is, for monoidal categories; see $[\mathrm{ML}])$ that this stronger condition is a consequence of the official definition, which is thus nothing but a finite (equational) axiomatization of the totality of all coherence conditions.

This suggests that, possibly, the right approach to the definition of weak $n$ category is to aim at formulating all coherence conditions at once, regardless the fact that this might give a very "theoretical" definition. It would then be a separate, and still very important, project to find a (hopefully) finite and concise set of coherence conditions that would be enough to imply all coherence conditions.

J. Baez and J. Dolan have produced a very interesting definition of "weak $n$ category" [BD2]. The similarity type of the Baez/Dolan $n$-category is radically larger than the usual one. In the usual $n$-categories, the domain and codomain of a $k+1$-cell are both $k$-cells; in a Baez/Dolan $n$-category, there are $k+1$-cells whose domains are arbitrary "pasting diagrams" of $k$-cells, not the composites of the latter. In addition, the coherence conditions are replaced, and the combinatorial complexity eliminated, by the requirement of the existence of cells satisfying certain universal properties (this phenomenon is well-known from Grothendieck's concept of fibration, relative to the concept of pseudo-functor; see [SGA1]).

The Baez/Dolan definition for $n=2$ can be shown to be closely related to the concept of "saturated anabicategory" mentioned above. However, Baez and Dolan do not use the ana-versions of morphisms, in particular, of "functor".

As I mentioned in the introduction, the Baez/Dolan definition is only a beginning, albeit a very promising one. Among others, the definition of the Baez/Dolan $n+1$-category of all $n$-categories is still missing. I believe that thinking in the spirit of anafunctors will help give the latter definition.

\section{Dependent types}

Dependent types are familiar from [M-L] and [C]; there is a further extensive literature of them. Their use for first-order logic seems to be new, however. First Order Logic with Dependent Sorts (FOLDS; I use "sort" in place of "type") is a variant of ordinary First Order Logic (FOL); the basic metatheory of FOLDS is an extension (generalization) of that of FOL.

A similarity type, or vocabulary, for FOLDS is a one-way category; a small category $\mathbf{L}$ is one-way if it has the following two properties:

(i) $\mathbf{L}$ has finite fan-out: for any $K \in \mathbf{L}$, there are only finitely many arrows with domain equal to $K$. 
(ii) $\mathbf{L}$ is reverse well-founded: there is no infinite sequence $\left\langle K_{n} \stackrel{f_{n}}{\longrightarrow} K_{n+1}\right\rangle_{n \in N}$ of composable proper (non-identity) arrows $\left(f_{n} \neq 1_{K_{n}}\right)$.

Immediate consequences of the definition are the following:

(iii) $\mathbf{L}$ is skeletal: any two isomorphic objects are identical;

(iv) the only arrow from an object to itself is the identity;

and in fact, as a consequence of (iii) and (iv),

(v) $\mathbf{L}$ has no circuit of positive finite length consisting of proper arrows; there is no $<K_{n} \stackrel{f_{n}}{\longrightarrow} K_{n+1}>_{n<N}$ with $N \in N, K_{N}=K_{0}$, and $f_{n} \neq 1_{K_{n}}(n<N)$.

It is easy to see that a small category is one-way iff it satisfies (i) and (v); and if the category is finite, then it is one-way iff it satisfies (iii) and (iv).

If $\mathbf{L}$ is a one-way category, the set $O b(\mathbf{L})$ of objects is partitioned as in

$$
O b(\mathbf{L})=\bigcup_{i<\ell} \mathbf{L}_{i}
$$

into non-empty levels $\mathbf{L}_{i}$, for $i<\ell, \ell$ the height of $\mathbf{L}, \ell \leq \omega$, such that $\mathbf{L}_{0}$ consist of the objects $A$ for which there is no proper arrow with domain $A$, and such that, for $i>0, \mathbf{L}_{i}$ consists of those objects $A$ for which all proper arrows $A \rightarrow B$ have $B \in \mathbf{L}_{<i}=\bigcup_{j<i} \mathbf{L}_{j}$, and there is at least one proper arrow $A \rightarrow B$ with $B \in \mathbf{L}_{i-1}$. All proper arrows go from a level to a lower level. Of course, the height of a finite one-way category is finite.

For $\mathbf{L}$ a one-way category, an $\mathbf{L}$-structure is a functor $\mathbf{L} \rightarrow$ Set.

There is a way of introducing FOLDS that follows Martin-Löf's idea of dependent types closely, without any preconceptions about similarity types; this is then seen to lead precisely to the notion of similarity type we just introduced without prior motivation (see [M3]).

The recognition of role of finite one-way categories in syntax is due to F. W. Lawvere, who pointed out their role in connection with the sketch-syntax of [M1]. Their role in FOLDS is related to their role in the sketch-syntax. I should note that the use of infinite one-way categories, in the sense used here, is essential (see below). Here is an example for a FOLDS similarity type, called $\mathbf{L}_{\text {cat }}$ : 


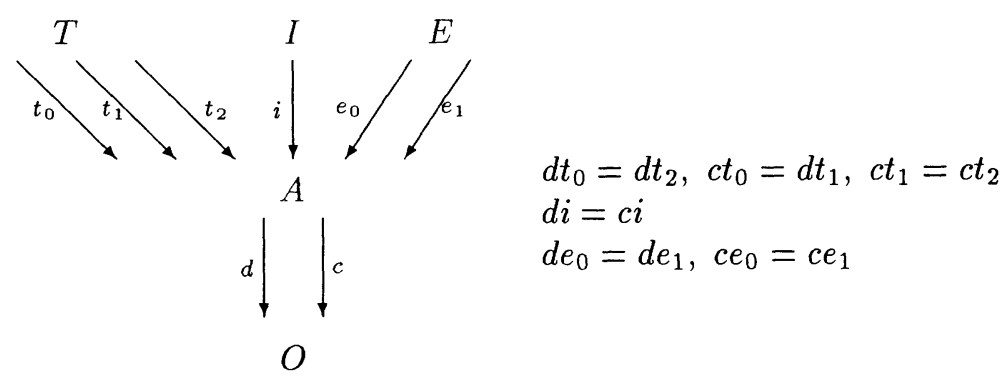

The proper arrows of $\mathbf{L}_{c a t}$ are the arrows shown, and their composites; the equalities shown identify some of these composites.

Note that any small category $\mathbf{C}$ gives rise to an $\mathbf{L}_{c a t}$-structure

$$
M[\mathbf{C}]=M: \mathbf{L}_{c a t} \rightarrow \text { Set }
$$

in a natural way. $M(O)$ is the set of objects of $\mathbf{C} ; M(A)$ is the set of arrows; $M(d)$ and $M(c)$ map an arrow to its domain and codomain, respectively. $M(T)$ is the set of commutative triangles, i.e., tuples

$$
(X, Y, Z, f: X \rightarrow Y, g: Y \rightarrow Z, h: X \rightarrow Z)
$$

such that $h=g f$. In other words, the elements of $M(T)$ are commutative diagrams of the form

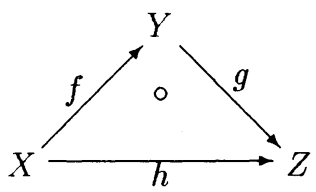

$M(I)$ is the set of identity arrows, $M(E)$ is the set of pairs $(f, f)$ of equal arrows. With $\tau$ standing for $(1), M\left(t_{1}\right)(\tau)=f, M\left(t_{2}\right)(\tau)=g, M\left(t_{3}\right)(\tau)=f$. The rest of the definition of $M$ should be clear. Note that $M$ is indeed a functor.

Of course, not every $\mathbf{L}_{c a t}$-structure is a category. On the other hand, for two categories construed as $\mathbf{L}_{c a t}$-structures, a natural transformation from one to the other is precisely the same as a functor from one category to the other.

Let $\mathbf{L}$ be a one-way category; we fix $\mathbf{L}$ for a while. The objects of $\mathbf{L}$ are called $k i n d s$. Let us write $K_{p}$ for $\operatorname{dom}(p)(p \in \operatorname{Arr}(\mathbf{L}))$. We use the notation $K \mid \mathbf{L}$ for the set of all proper arrows $p: K \rightarrow K_{p}$ with domain $K$. The set $K \mid \mathbf{L}$ will figure as the arity of the symbol $K$. In particular, the ones with empty arity are exactly the level- 0 kinds.

We are going to define what sorts are, and what variables of a given sort are; we will write $x: X$ to denote that the variable $x$ is of sort $X$. Every sort will be of the form $K\left(\left\langle x_{p}\right\rangle_{p \in K \mid \mathrm{L}}\right)$, with $K$ a kind (the displayed sort is then said to 
be of the kind $K$ ), with variables $x_{p}$ indexed by the elements $p$ of the arity $K \mid \mathbf{L}$ of $K$; additional conditions will have to be satisfied. Let $k \in N$, and suppose we have defined sorts of kinds on levels less than $k$, and variables of such sorts. Then, for a kind $K$ on level $k, K\left(\left\langle x_{p}\right\rangle_{p \in K \mid \mathbf{L}}\right)$ is a sort (of the kind $K$ ) iff for each $p \in K \mid \mathbf{L}$, we have $x_{p}: K_{p}\left(\left\langle y_{p, q}\right\rangle_{q \in K_{p} \mid \mathbf{L}}\right)$ with $p: K \rightarrow K_{p}$ (note that $K_{p}$ is on a lower level than $K$ ), and for every $q \in K_{p} \mid \mathbf{L}, y_{p, q}=x_{q p}$ :

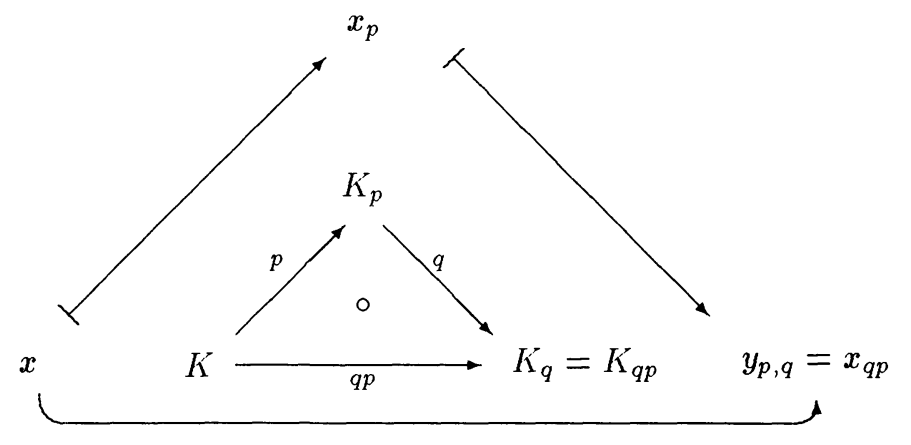

For every sort $X=K\left(\left\langle x_{p}\right\rangle_{p \in K \mid \mathbf{L}}\right)$ thus specified, we declare certain symbols as variables of sort $X$; the only important things about this declaration are that (i) variables of sort $X$ have to be new, so that every variable uniquely determines its own sort, and that (ii) there are enough (infinitely many) variables of each sort.

Note that every variable "carries" its own sort with it. This is in contrast with the practice of most of the relevant literature (see e.g. [C]), where variables are "locally" declared to be of certain definite sorts, but by themselves, they do not carry sort information. For a sort

$$
X=K\left(<x_{p}>_{p \in K \mid \mathrm{K}}\right), \operatorname{Var}(X) \underset{\text { def }}{=}\left\{x_{p}: p \in K \mid \mathbf{K}\right\} ;
$$

and if $x: X, D e p(x) \underset{\text { def }}{=} \operatorname{Var}(X) ; x$ depends on the variables in $\operatorname{Dep}(x)$.

Let $\mathbf{L}=\mathbf{L}_{\text {cat }}$. Since $O \mid \mathbf{L}$ is empty, $O(\emptyset)$, with $\emptyset$ the empty sequence of variables, is a sort; we write simply $O$. We have $A \mid \mathbf{L}=\{d, c\}$; the sorts of the kind $A$ are of the form $A(X, Y)$, with $X, Y: O$; here, $X$ is indexed by $d, Y$ by $c$.

$T \mid \mathbf{L}=\left\{f_{1}, f_{2}, f_{3}, t_{1}, t_{2}, t_{3}\right\}$, with $f_{1}=d t_{1}=d t_{3}, f_{2}=c t_{1}=d t_{2}, f_{3}=c t_{2}=c t_{3}$. A sort of the kind $T$ will have the form $T(X, Y, Z, f, g, h)$; applying the condition in the definition with $p=t_{1}$, we get that $f: A(X, Y)$ must be the case. In summary, the variables in $T(X, Y, Z, f, g, h)$ have to line up as in

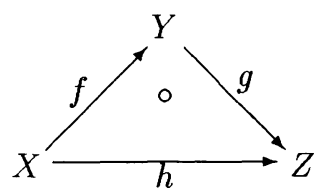


We define formulas $\varphi$ and the set $\operatorname{Var}(\varphi)$ of the free variables of $\varphi$ by a simultaneous induction.

The symbols $\mathbf{t}$ ("true"), $\mathbf{f}$ ("false") are formulas; $\operatorname{Var}(\mathbf{t})=\operatorname{Var}(\mathbf{f})=\emptyset$.

The sentential connectives $\wedge, \vee, \rightarrow, \neg, \leftrightarrow$ can be applied in an unlimited manner; $\operatorname{Var}()$ for the compound formulas formed using connectives is defined in the expected way; e.g., $\operatorname{Var}(\varphi \wedge \psi)=\operatorname{Var}(\varphi) \cup \operatorname{Var}(\psi)$.

Suppose $\varphi$ is a formula, $x$ is a variable such that there is no $y \in \operatorname{Var}(\varphi)$ with $x \in \operatorname{Dep}(y)$. Suppose $x: X$. Then $\forall x: X . \varphi, \exists x: X . \varphi$ are formulas;

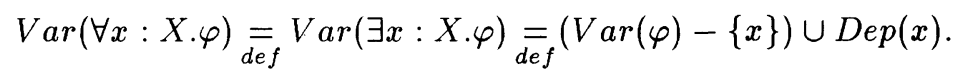

All formulas are obtained as described.

Here is an example of a sentence (formula without free variables) over $\mathbf{L}_{c a t}$ :

$\forall X: O . \forall Y: O . \forall Z: O . \forall f: A(X, Y) . \forall g: A(Y, Z) . \exists h: A(X, Z) . \exists t: T(X, Y, Z, f, g, h) . \mathbf{t}$

The sentence, referred to below as (2), expresses the existence of the composite $h: X \rightarrow Z$ of composable arrows $f: X \rightarrow Y, g, Y \rightarrow Z$.

The sorts are interpreted in structures as certain sets. Let $K$ be a kind, $M$ an $\mathbf{L}$ structure. By $M[K]$ we mean the set of all tuples $\left\langle a_{p}\right\rangle_{p \in K \mid \mathbf{L}}$ with $a_{p} \in M\left(K_{p}\right)$ (where $p: K \rightarrow K_{p}$ ), and such that for every $p \in K \mid \mathbf{L}$ and $q \in K_{p} \mid \mathbf{L}, M(q)\left(a_{p}\right)=$ $a_{q p}$ :

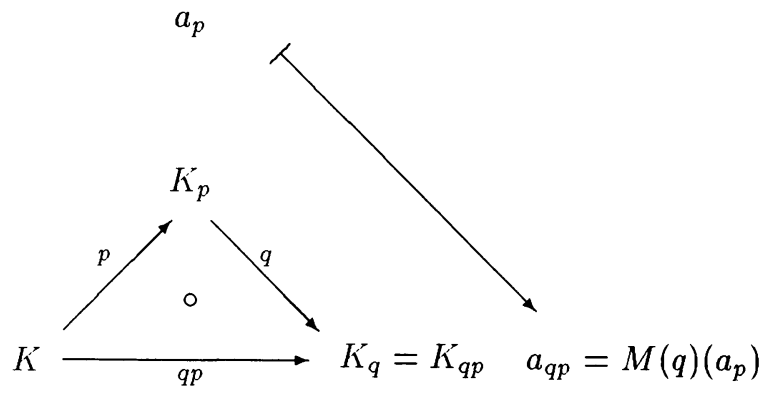

The elements of the set $M[K]$ are called contexts for $K$ in $M$.

For instance, for $\mathbf{L}=\mathbf{L}_{\text {cat }}$, when $K=A$ and $M$ is $M[\mathbf{C}]$ for a category $\mathbf{C}$, then $M[A]$ is the set of pairs $(X, Y)$ of objects; when $K=T, M[T]$ is the set of all not necessarily commutative triangles in $\mathbf{C}$. 


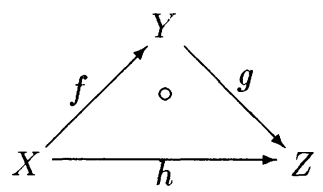

The set $M(K)$ is "fibered over" $M[K] ; M(K)$ is the disjoint union of sets $M K(\mathbf{a})$, one for each $\mathbf{a}=\left\langle a_{p}\right\rangle_{p \in K \mid \mathbf{L}} \in M[K]$;

$$
M K(\mathbf{a}) \underset{\text { def }}{=}\left\{a \in M(K):(M p)(a)=a_{p} \text { for all } p \in K \mid \mathbf{L}\right\},
$$

the fiber over a (it is clear that for any $a \in M(K)$,

$$
\left.\mathbf{a} \underset{\text { def }}{=}<(M p)(a)>_{p \in K \mid \mathbf{L}} \in M[K]\right) .
$$

Let $X=K\left(\left\langle x_{p}\right\rangle_{p \in K \mid \mathbf{L}}\right)$ be a sort. An interpretation of $X$ in $M$ is given by a context $\mathbf{a}=\left\langle a_{p}\right\rangle_{p \in K \mid \mathrm{L}} \in M[K]$, with $a_{p}$ assigned to $x_{p}$, which means the additional condition $a_{p}=a_{p^{\prime}}$, every time $x_{p}=x_{p^{\prime}},\left(p, p^{\prime} \in K \mid \mathbf{L}\right)$; the interpretation itself is the fiber $M K(\mathbf{a})$ over $\mathbf{a}$.

Returning to the example started above, the interpretation of $A(X, Y)$ is hom $\mathbf{C}$ $(A, B)$ when $A, B$ are objects assigned to the variables $X, Y$ respectively. The interpretation of the sort $T(X, Y, Z, f, g, h)$ must be given by a (not necessarily commutative) diagram of the shape

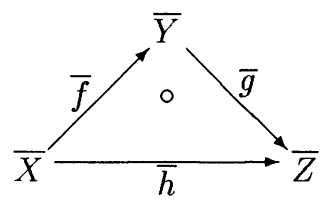

in $\mathbf{C}$, and of course, if, say, $X$ is the same variable as $Y$, then the object $\bar{X}$ must be the same as $\bar{Y}$. The interpretation itself is a singleton set if the displayed diagram commutes, and the empty set otherwise.

For the full definition of the semantics of FOLDS, we need the concept of context (of variables). A context is a finite set $\mathcal{Y}$ of variables such that, for all $y \in \mathcal{Y}$ we have that $\operatorname{Dep}(y) \subset \mathcal{Y}$.

For instance, in the case $\mathbf{L}=\mathbf{L}_{c a t}$, the set $\{X, Y, Z, f, g, h, t\}$, under the conditions

$$
X: O, Y: O, Z: O, f: A(X, Y), g: A(Y, Z), z: A(X, Z), t: T(X, Y, Z, f, g, h),
$$
is a context. 
Note that for any formula $\varphi, \operatorname{Var}(\varphi)$ is a context.

Let $\mathcal{Y}$ be a context, and $M$ an $\mathbf{L}$-structure. We define the set $M[\mathcal{Y}]$, the set of (legitimate) valuations of $\mathcal{Y}$ in $M$.

For a variable $y \in \mathcal{Y}$, let us display the sort of $y$ in the notation

$$
y: K_{y}\left(<x_{y, p}>_{p \in K_{y} \mid \mathbf{L}}\right) .
$$

We define $M[\mathcal{Y}] \underset{\text { def }}{=}$

$\left\{<a_{y}>_{y \in \mathcal{Y}} \in \prod_{y \in \mathcal{Y}} M\left(K_{y}\right):(M p)\left(a_{y}\right)=a_{z}\right.$ whenever $y \in \mathcal{Y}, p \in K_{y} \mid \mathbf{L}$ and $\left.z=x_{y, p}\right\}$.

The definition says that the elements of $M[\mathcal{Y}]$ are compatible valuations of the variables in $\mathcal{Y}$, where "compatibility" refers to the fact that if two variables $y$ and $z$ from $\mathcal{Y}$ are in a relation of dependence, $z \in D e p(y)$, in a particular way given by the "place" $p$ of the kind $K_{y}$ (that is, $z=x_{y, p}$ ), then the corresponding elements of the family have to be related by $M(p)$.

By recursion on the complexity of the formula $\varphi$, we define $M[\mathcal{Y}: \varphi]$, the interpretation of $\varphi$ in $M$ in the context $\mathcal{Y}$, whenever $\mathcal{Y}$ is a context such that $\operatorname{Var}(\varphi) \subset \mathcal{Y}$; we will have that $M[\mathcal{Y}: \varphi] \subset M[\mathcal{Y}]$.

$$
\begin{gathered}
M[\mathcal{Y}: \mathbf{t}] \underset{\text { def }}{=} M[\mathcal{Y}] ; \\
M[\mathcal{Y}: \mathbf{f}] \underset{\text { def }}{=\emptyset} .
\end{gathered}
$$

For the propositional connectives, the clauses are the expected ones; e.g.,

$$
\begin{gathered}
<a_{y}>_{y \in \mathcal{Y}} \in M[\mathcal{Y}: \psi \wedge \theta] \underset{d e f}{\Longleftrightarrow} \\
<a_{y}>_{y \in \mathcal{Y}} \in M[\mathcal{Y}: \psi] \text { and }\left\langle a_{y}>_{y \in \mathcal{Y}} \in M[\mathcal{Y}: \theta] .\right.
\end{gathered}
$$

The interpretation of formulas $\forall x: X . \psi, \exists x: X . \psi$ will be according to the readings "for all $x$ in $X, \psi$ ", and "there is $x$ in $X$ such that $\psi$ ". Thus, quantification in FOLDS is a relativized quantification. Here, the sort $X$ is interpreted as a set according to what was said above. For the precise clause, we need a bit more notation.

Let $M, \mathcal{Y}$ and $\mathbf{a}=\left\langle a_{y}\right\rangle_{y \in \mathcal{Y}} \in M[\mathcal{Y}]$ be as above; assume

$$
\operatorname{Var}(\forall x: X . \psi)=\operatorname{Var}(\exists x: X . \psi)=(\operatorname{Var}(\varphi)-\{x\}) \cup \operatorname{Dep}(x) \subset \mathcal{Y} .
$$

Part of $\mathcal{Y}$ may be discarded. Let $\mathcal{Y}^{\prime}=\mathcal{Y}-\{x\}-\{y \in \mathcal{Y}: x \in D e p(y)\}$. Then, still, $\mathcal{Y}^{\prime}$ is a context, and $\operatorname{Var}(\forall x \psi) \subset \mathcal{Y}^{\prime}$ (the reason is that, since $\forall x: X . \psi$ is well-formed, if $y \in \operatorname{Var}(\psi)$, then $x \notin \operatorname{Dep}(y))$. Also, $\mathcal{Y}^{\prime} \cup\{x\}$ is a context, and $\operatorname{Var}(\psi) \subset \mathcal{Y}^{\prime} \cup\{x\}$. 
Let $\left.X=K\left(<x_{p}\right\rangle_{p \in K \mid \mathrm{L}}\right)$. Each $x_{p} \in \mathcal{Y}^{\prime}$, so $\tilde{a}=\left\langle a_{x_{p}}\right\rangle_{p \in K \mid \mathrm{L}}$ is defined, and as easily seen, $\tilde{a} \in M[K]$. Therefore, we have the fiber $M K(\tilde{a})$ of $M(K)$.

Let $\mathbf{a}=\left\langle a_{y}\right\rangle_{y \in \mathcal{Y}} \in M[\mathcal{Y}]$. Define $\mathbf{a}^{\prime}=\left\langle a_{y}\right\rangle_{y \in \mathcal{Y}^{\prime}}$. For any $a \in M K(\tilde{a})$, let $\mathbf{a}^{\prime}[a / x]$ be $\left\langle b_{y}\right\rangle_{y \in \mathcal{Y}^{\prime} \cup\{x\}}$ for which $b_{y}=a_{y}$ when $y \in \mathcal{Y}^{\prime}$, and $b_{x}=a$. It is immediately seen that $\mathbf{a}^{\prime}[a / x] \in M\left[\mathcal{Y}^{\prime} \cup\{x\}\right]$. We define

$$
\mathbf{a} \in M[\mathcal{Y}: \forall x: X . \psi] \Leftrightarrow \mathbf{a}^{\prime}[a / x] \in M\left[\mathcal{Y}^{\prime} \cup\{x\}: \psi\right] \text { for all } a \in M K(\tilde{a}),
$$

and

$$
\mathbf{a} \in M[\mathcal{Y}: \exists x: X . \psi] \Leftrightarrow \mathbf{a}^{\prime}[a / x] \in M\left[\mathcal{Y}^{\prime} \cup\{x\}: \psi\right] \text { for some } a \in M K(\tilde{a}) .
$$

This completes the definition of the standard, Set-valued semantics of FOLDS.

As usual, we also write $M \vDash \varphi[\mathbf{a}]$ for $\mathbf{a} \in M[\mathcal{Y}: \varphi]$.

Returning to the example of $\mathbf{L}_{c a t}$, the reader will easily write down all axioms for "category" in the form of sentences over $\mathbf{L}_{c a t}$; the sentence $(2)$ above is an example. Let us call the resulting finite set of sentences $\Sigma_{c a t}$. Since equality is not treated as "logical", $\Sigma_{c a t}$ includes axioms concerning equality, the kind $E$. It will almost, but not quite, be the case that an $\mathbf{L}_{\text {cat }}$-structure $M$ satisfies $\Sigma_{c a t}$ iff it is of the form $M=M[\mathbf{C}]$ for a category $\mathbf{C}$; for explanation, see below.

\section{Formal systems}

FOLDS may be regarded as a restricted form of ordinary first order logic. Given a one-way category $\mathbf{L}$, we may consider the multi-sorted first order language with sorts the objects of $\mathbf{L}$, with unary sorted operation symbols the arrows of $\mathbf{L}$, and with equality predicates, one for each sort; let us refer to this language as First Order Logic (FOL) over $\mathbf{L}$. The formulas of FOLDS over $\mathbf{L}$ can obviously be translated into FOL over $\mathbf{L}$. This consideration will immediately imply the fact that the compactness theorem, a kind of abstract completeness, holds for FOLDS.

We have completeness theorems of appropriate formal systems for FOLDS. Completeness is stronger than compactness; it is also essential for the purpose of adopting FOLDS as a language for an axiomatic system.

We have the classical and the intuitionistic versions of FOLDS. The third version, coherent logic, is a proper part of both classical and intuitionistic logic. Categorical logic, mainly in the pioneering work of Andre Joyal, has shown the fundamental theoretical role of coherent logic in relation to both classical and intuitionistic logic, for example, in connection with completeness theorems (see [MR1]). The coherent fragment of FOLDS continues to play a basic role. 
In [M3], I worked out the completeness theorems for systems in a format following [LS]. This format is the one that fits best a categorical framework for the metatheory of FOLDS.

The Lambek-style system employ entailments. An entailment is an entity of the form $\theta \underset{\mathcal{X}}{\Rightarrow} \sigma$, with $\theta$ and $\sigma$ formulas, and $\mathcal{X}$ a context such that $\operatorname{Var}(\theta) \cup \operatorname{Var}(\sigma) \subset$ $\mathcal{X}$. The intended meaning of the entailment $\theta \underset{\mathcal{X}}{ } \sigma$ is that of $\forall \mathcal{X}(\theta \rightarrow \sigma)$, where $\forall \mathcal{X}$ is the string of universal quantifiers $\forall x$, with $x$ ranging over all $x \in \mathcal{X}$ in an appropriate order. The formal system consists of rules of inference, in any instance of which zero, one or two entailments, the premisses, yield another, the conclusion. (The inference rules with zero premisses are axiom schemes.) We have systems for each of coherent, intuitionistic and classical logic. We are not going to describe the systems in full; all the less since they present no surprises in comparison to ordinary first order logic; see [M3] for the details. The most characteristic rules are the quantifier rules; the ones for the universal quantifier are as follows:

$$
\frac{\theta \overrightarrow{\overline{\mathcal{X} \cup x\}}} \sigma}{\overline{\overrightarrow{\underline{X}} \forall x: X . \sigma}}
$$

The display contains two rules: one is the upper entailment implying the lower one, the other vice versa. The upper entailment is assumed to be well-formed; in particular, $\mathcal{X}$ and $\mathcal{X} \cup\{x\}$ are both contexts, and $\operatorname{Var}(\sigma)$ is a subset of $\mathcal{X} \cup\{x\}$; also $x \notin \mathcal{X}$. It is interesting to note that these facts imply that $\forall x: X . \sigma$ is well-formed.

A theory $T$ in FOLDS is a pair $T=(\mathbf{L}, \Sigma)$, where $\mathbf{L}$ is a one-way category, and $\Sigma$ is a set of "axiom" entailments over $\mathbf{L}$. For an entailment $\alpha$ over $\mathbf{L}$, we write $T \vdash \alpha$, and say that $\alpha$ is deducible from $T$, if $\alpha$ can be obtained from the axioms in $\Sigma$ by repeated use of the rules of inference. On the other hand, $T \vDash \alpha$ if every (ordinary, set-valued) model of $T$ is a model of $\alpha$ according to the intended meaning of entailments.

The (Gödel) completeness theorems for the coherent fragment, and for classical version, of FOLDS say that $T \vdash \alpha$ and $T \vDash \alpha$ are equivalent, for any $T$ and $\alpha$ within the respective logic. Completeness for the classical system is a consequence of that for the coherent fragment, just like for ordinary first-order logic (see [MR1]).

Besides the classical, intuitionistic and coherent versions of FOLDS, there is a fourth that is important: this is the regular fragment. In this, the only formulas (regular formulas) are the ones built of $t, \wedge$ and $\exists$. Regular entailments are $\theta \underset{\mathcal{X}}{\Rightarrow} \sigma$ for regular formulas $\theta, \sigma$.A regular theory is one whose axioms are regular entailments. The regular fragment of FOLDS is the one which comes closest to the essentially algebraic fragment [Co] of first order logic, which has $\exists$ restricted 
to the unique existential quantifier. In FOLDS, the uniqueness condition for $\exists$ is inappropriate in most situations, because of the lack of equality. All the significant types of categorical structure are axiomatized in the regular fragment, provided we employ the appropriate similarity type. For instance, the concept of "category with binary product" can be axiomatized in FOLDS over $\mathbf{L}_{c a t}$, although not in regular FOLDS. But if we add a new kind to $\mathbf{L}_{c a t}$, one for product diagrams, with appropriate arrows, the concept becomes axiomatizable in regular FOLDS. Regular FOLDS is closely related to sketch-syntax of [M1].

Completeness theorems for intuitionistic logic employ "non-standard" semantics. For any category $\mathbf{S}$, the concept of $\mathbf{S}$-valued $\mathbf{L}$-structure is automatically available, in the form of functors $\mathbf{L} \rightarrow \mathbf{S}$. In order for this concept to be of value, $\mathbf{S}$ needs to have a certain amount of structure; the minimum is $\mathbf{S}$ being a regular category (see [B]). In applications, $\mathbf{S}$ is usually much more than regular. For the modeling of intuitionistic logic, $\mathbf{S}$ is taken to be a Grothendieck topos. In particular, for Kripke-style modeling, $\mathbf{S}$ is a category of the form $\mathrm{Set}^{\mathbf{C}}$, for some small category (e.g., a poset). For modeling in $H$-valued sets, for $H$ a complete Heyting algebra, $\mathbf{S}$ is the topos of $H$-valued sets, or equivalently, the topos of sheaves over $H$.

S-valued semantics uses the interpretation of formulas in FOLDS in the category $\mathbf{S}$. This is based on the available structure of $\mathbf{S}$; if $\mathbf{S}$ is a topos, all of FOLDS can be interpreted in $\mathbf{S}$. This interpretation will be fairly clear to readers familiar with categorical semantics; see e.g. [MR1].

For the model theory of intuitionistic FOLDS, and indeed, for the metatheory of FOLDS in general, including the proof of the Gödel completeness theorems, it is good to put FOLDS into a framework of fibrations (see [Gr], [M7]). A theory in FOLDS becomes a quantificational fibration with a base category having finite limits (a terminal object, and pullbacks), with a class of distinguished arrows in the base category stable under pullback, with an appropriate structure in the fibers (corresponding to the propositional connectives), and with quantification along the distinguished arrows only in the form of adjoints to substitutionfunctors between the fibers. Upon the said identification of theories with certain categorical structures (fibrations), an interpretation of one theory in another, and models of theories in a category $\mathbf{S}$, become morphisms of the appropriate categorical structures (we associate with the category $\mathbf{S}$ a fibration in a standard way; see [M7]).

The said fibrational framework represents a generalization of FOLDS, with the result that the general theory can be applied to other things, notably the concept of bisimulation in theoretical computer science.

\section{Equivalence in FOLDS}

The main pont about FOLDS is that it comes with an appropriate notion of structural equality. For each similarity type $\mathbf{L}$ for FOLDS, we have the concept 
of $\mathbf{L}$-equivalence of $\mathbf{L}$-structures. Properties expressible in FOLDS over $\mathbf{L}$ are invariant under $\mathbf{L}$-equivalence. There is a strong converse to the last statement, which shows that the syntax of FOLDS and L-equivalence are precisely matched. The issues of invariance are addressed in the next section; in this section, the meaning of $\mathbf{L}$-equivalence is explored.

Let $M, P$ be $\mathbf{L}$-structures, $h: P \rightarrow M$ a natural transformation. $h$ induces the "componentwise" map $h_{[K]}: P[K] \rightarrow M[K]$; and for any $\mathbf{x} \in P[K]$, and for $\mathbf{a}=h_{[K]}(\mathbf{x})$, the map

$$
h_{\mathbf{x}}: P K(\mathbf{x}) \longrightarrow M K(\mathbf{a})
$$

between the corresponding fibers. $h$ is called fiberwise surjective if $h_{\mathbf{x}}$ is surjective for all $\mathbf{x} \in P[K]$.

Let $M, N$ be L-structures. An L-equivalence of $M$ and $N$ is a triple $(P, h, k)$ where

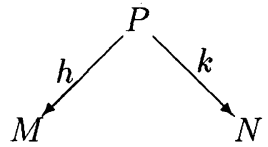

and both $h$ and $k$ are fiberwise surjective maps; for this, we write

$$
(P, h, k): M \simeq_{\mathbf{L}} N .
$$

$M$ and $N$ are L-equivalent, $M \simeq_{\mathbf{L}} N$, if there exists an $\mathbf{L}$-equivalence between them.

It is important, e.g., for FOLDS in an "intuitionistic (constructive) world", that the concept of $\mathbf{L}$-equivalence has a natural extension to $\mathbf{S}$-valued structures, for any regular category $\mathbf{S}$. When $M: \mathbf{L} \rightarrow \mathbf{S}$, and $K \in \mathbf{L}$, there is a natural definition of $M[K]$ the object of $K$-contexts in $M$, as a certain finite limit in $\mathbf{L}$. Then $h: P \rightarrow M$ a natural transformation of $\mathbf{S}$-valued $\mathbf{L}$-structures, is said to be fiberwise surjective if in the induced commutative diagram

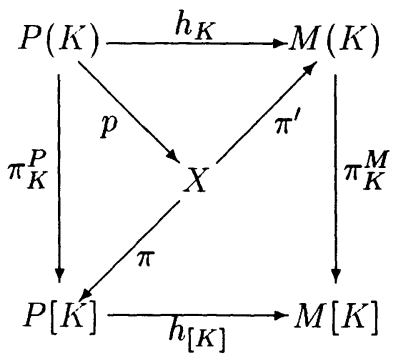

in which $X$ is a pullback with projections $\pi$ and $\pi^{\prime}$, the arrow $p$ is surjective, that is, a regular epimorphism (one says for this that the outside rectangle is a quasi-pullback). 
L-equivalence is an equivalence relation. Reflexivity and symmetry are clear. For transitivity, we use composition of $\mathbf{L}$-equivalences as spans; that is, given the equivalences $(Q, h, k): M \simeq_{\mathrm{L}} N,(R, \ell, m): N \simeq_{\mathrm{L}} P$, we construct the pullback $S$ with projections as in

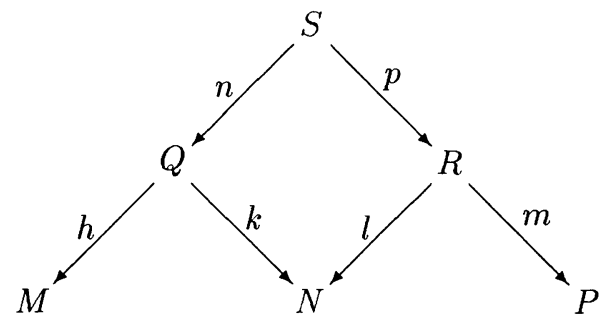

the desired composite equivalence is $(S, h n, m p): M \simeq_{\mathbf{L}} P$.

To understand L-equivalence, we should note that, for Set-valued models at least, a simplified concept, that of a "relational $\mathbf{L}$-equivalence", suffices. The L-equivalence $(P, h, k): M \simeq_{\mathbf{L}} N$ is relational if the arrows $h$ and $k$ are jointly monic. Equivalently, a relational equivalence is given by a family $\rho=\left\langle\rho_{K}\right\rangle_{K \in \mathbf{L}}$ of relations $\rho_{K} \subset M K \times N K$ such that, with

$$
\mathbf{a} \rho_{[K]} \mathbf{b} \underset{\text { def }}{\Longleftrightarrow} \forall p \in K \mid \mathbf{L} \cdot a_{p} \rho_{K_{p}} b_{p},
$$

for $\mathbf{a}=\left\langle a_{p}>_{p \in K \mid \mathbf{L}} \in M[K], \mathbf{b}=\left\langle b_{p}\right\rangle_{p \in K \mid \mathbf{L}} \in N[K]\right.$, the following hold:

(i) For any $p: K \rightarrow K_{p}, a \in M K, b \in N K$

$$
a \rho_{K} b \Rightarrow(M p)(a) \rho_{K_{p}}(N p)(b) .
$$

(ii) For any $K \in \mathbf{K}, \mathbf{a} \in M[K], \mathbf{b} \in N[K]$,

$$
\begin{aligned}
& \mathbf{a} \rho_{[K]} \mathbf{b} \& a \in M K(\mathbf{a}) \Rightarrow \exists b \in N K(\mathbf{b}) \cdot a \rho_{K} b . \\
& \mathbf{a} \rho_{[K]} \mathbf{b} \& b \in N K(\mathbf{b}) \Rightarrow \exists a \in M K(\mathbf{a}) \cdot a \rho_{K} b .
\end{aligned}
$$

Let us take the example of the similarity type $L$ of one-sorted relational structures (essentially, the only one that standard model theory deals with). $L$ can be construed as a one-way category $\mathbf{L}$ with two levels, on the bottom level a single object, the "sort", and on level-1 the relation symbols, with a symbol for the equality relation added. From each relation symbol of arity $k$, there are $k$ arrows to the sort. Clearly, each $L$-structure can be construed as an $\mathbf{L}$-structure, although the correspondence is not bijective; the $L$-structures correspond to $\mathbf{L}$ structures in which the level-1 fibers are either empty or singletons, and in which the equality is real equality. Now, the point is that, for $\mathbf{L}$-structures coming from $L$-structures, relational $\mathbf{L}$-equivalences are the same as isomorphisms in the usual sense. 
In the clause (ii), there is a formal resemblance to the "back-and-forth" systems for equivalence in the logic $L_{\infty}$; see, e.g. [Ba]. As the previous paragraph shows, when we look at the simplest case, the notion of relational equivalence is not at all $L_{\infty \omega}$-equivalence; it is ordinary isomorphism. Nevertheless, the resemblance does lead to a unified view of back and forth properties and FOLDS equivalence, and to an interesting group of facts about infinitary first order logic with dependent sorts; see [M3].

Another concept one is reminded of by the above is the notion of bisimulation in theoretical computer science; see [MPW]. This connection is genuine. The more general version of the basic theory, with the quantificational fibrations at the basis, yields as special cases of FOLDS equivalence some of the concepts of bisimulation treated in the literature. In this context, the language of FOLDS is seen to generalize the Henessy-Milner language associated to bisimulation.

If two Set-valued $\mathbf{L}$-structures are $\mathbf{L}$-equivalent, then there is a relational equivalence between them. The proof of this uses Choice, which indicates that the concept of relational equivalence is not optimal. Indeed, it does not work when we pass to $\mathbf{S}$-structures for more general $\mathbf{S}$. In fact, it does not work even in the Set-valued case when we want to keep track of individual elements in the structures under the equivalence.

Let us consider the case $\mathbf{L}=\mathbf{L}_{\text {cat }}$. At the end of section 8, we mentioned the theory $T_{c a t}=\left(\mathbf{L}_{c a t}, \Sigma_{c a t}\right)$ whose models are almost the same as categories. The fact is that every model $M$ of $T_{\text {cat }}$ is $\mathbf{L}$-equivalent to $M[\mathbf{C}]$ for a category $\mathbf{C}$. The construction of $\mathbf{C}$ involves cutting down every non-empty level-2 fiber in $M$ to a singleton, and taking appropriate equivalence classes of equivalence relations induced by $E$. Indeed, the underlying facts here apply to general FOLDS similarity types. Since $\mathbf{L}$ - equivalence is treated as the structural equality for $\mathbf{L}$-structures, it is fair to say that $T_{c a t}$ axiomatizes the notion of category.

From now on, we will write $\mathbf{C}$ for $M[\mathbf{C}]$ as well; the context will clarify any potential ambiguity.

We have that, for categories $\mathbf{C}$ and $\mathbf{D}, \mathbf{L}$-equivalence coincides with ordinary equivalence of categories:

$$
\mathbf{C} \simeq \mathbf{D} \Leftrightarrow \mathbf{C} \simeq_{\mathrm{L}_{\text {cat }}} \text { D. }
$$

The construction from an ordinary equivalence to an $\mathbf{L}$-equivalence is a canonical one; it uses the anafunctor-saturation (see section 5) of the functors involved. In fact, it suffices to have a single equivalence functor $F: \mathbf{C} \rightarrow \mathbf{D}$, with the properties that it is fully faithful (induces bijections $\mathbf{C}\left(C, C^{\prime}\right) \rightarrow \mathbf{D}\left(F C, F C^{\prime}\right)$ ), and surjective on objects up to isomorphism; the saturation of $F$ will yield an $\mathbf{L}$ equivalence. Conversely, an $\mathbf{L}$-equivalence gives rise to a categorical equivalence by the use of the axiom of choice. 
The case of more complicated categorical concepts is more interesting; a passage to the ana-concepts is involved.

The simplest such structures are the "free-living" functors; two categories connected by a functor. The most well-known type of such a structure is fibration. In [MR2], G.E. Reyes and the author have invesitgated "free-living" functors with properties derived from universal properties as a basis for categorical formulations of versions of intuitionistic and modal logics; these "modal categories" (actually, functors) are not fibrations in general.

There is a concept of equivalence of functors as follow:

$F: \mathbf{X} \rightarrow \mathbf{A}$ and $G: \mathbf{Y} \rightarrow \mathbf{B}$ are equivalent, in notation $F \simeq G$, if there are equivalence-functors $E_{0}, E_{1}$ and an isomorphism $e$ as in the diagram

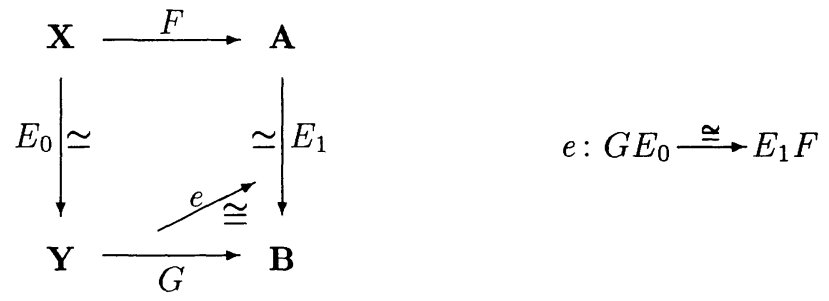

This notion is the combination of (the least equivalence relation containing) isomorphism of parallel functors, and equivalence of categories. In several places in the literature, it is recognized that this is the "right" notion of equivalence for functors. An early example of this is Kelly's paper [K] quoted before. When one has a theorem to the effect that a free-living functor of a certain kind can be presented, or constructed, in a certain way, then one expects that the result will be only equivalent to the original. This is the case when the theorem needs noncanonical choices in its proof. An example for this is the Uniform Sketchability Theorem of [MP].

The properties appearing in [MR2] of functors are all invariant under equivalence. The concept of fibration is not invariant under equivalence; however, restricted to fibrations, the additional properties of fibrations usually considered are.

The FOLDS similarity type to be considered in connection with functors is the following one-way category $\mathbf{L}_{\text {fun }}$ :

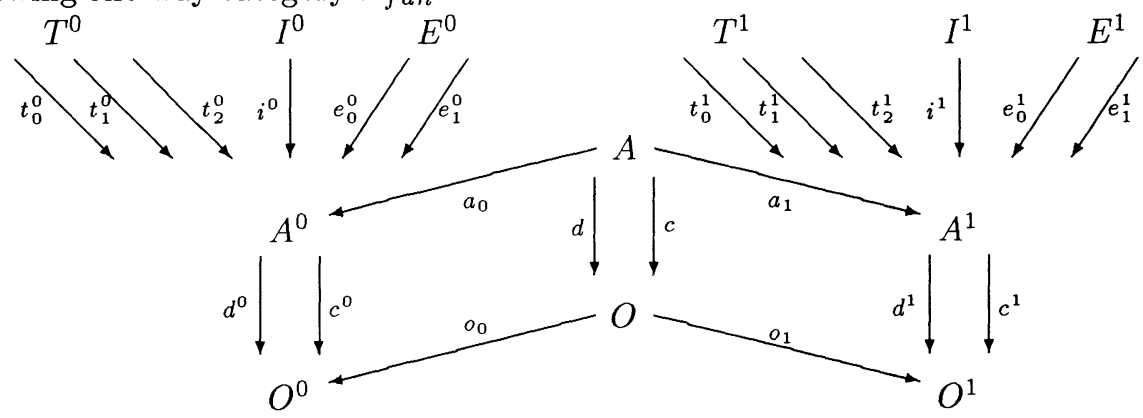


Here, we have two copies of $\mathbf{L}_{c a t}$; the rest is subject to the following equalities:

$$
d^{0} a_{0}=o_{0} d, c^{0} a_{0}=o_{0} c, d^{1} a_{1}=o_{1} d, c^{1} a_{1}=o_{1} c .
$$

Every free-living functor is, in a natural way, a functor $\mathbf{L}_{f u n} \rightarrow$ Set. For instance, $O$ and $A$ are interpreted as the graphs of the object-function and the arrow function of the given functor.

Now, we do not have the fact that the equivalences in the usual categorical sense and the FOLDS sense are the same as in the case of categories. However, every functor $F: \mathbf{X} \rightarrow \mathbf{A}$ gives rise to the saturated anafunctor $F^{\#}: \mathbf{X} \stackrel{a}{\longrightarrow} \mathbf{A}$ mentioned at the end of section 5. $F^{\#}$ is also an $\mathbf{L}_{f u n}$-structure. $F^{\#}$ interprets the two copies of $\mathbf{L}_{c a t}$ in $\mathbf{L}_{f u n}$ just as $F$ does.

$$
F^{\#}(A) \underset{\text { def }}{=}\{(X, A, \mu): X \in O b(\mathbf{X}), A \in O b(\mathbf{A}), \mu: F(X) \stackrel{\simeq}{\longrightarrow} A\},
$$

with $(X, A, \mu) \stackrel{F^{\#}{ }_{o_{0}}}{\mapsto} X,(X, A, \mu) \stackrel{F^{\#} o_{o_{1}}}{\mapsto} A$; and

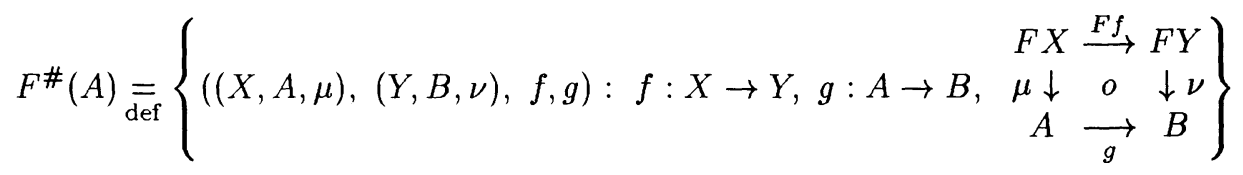

In other words, $\left|F^{\#}\right|(X, A)=\operatorname{Iso}(F X, A)=\{\mu \in \operatorname{hom}(F X, A): \mu$ is iso $\}$, and, with the notation used for $F^{\#}(A), F_{\mu, \nu}(f)=g$ determined by the commutative square. The displayed item in $F^{\#}(A)$ is mapped to $f$ by $F^{\#}\left(a_{0}\right)$, and to $g$ by $F^{\#}\left(a_{1}\right)$. One can prove (using the Axiom of Choice) that the saturated anafunctors are, up to isomorphism as $\mathbf{L}$-structures, the same as the $F^{\#}$ for functors $F$.

Now, the main fact is that with $\mathbf{L}=\mathbf{L}_{f u n}$, for any functors $F$ and $G$,

$$
F \simeq G \Leftrightarrow F^{\#} \simeq_{\mathrm{L}_{f u n}} G^{\#} .
$$

We may look at this fact as saying that the passage to the saturation "straightens out" the somewhat ad hoc notion of equivalence for functors into something that follows a general logical pattern.

There is a similar situation in the case of the notion of bicategory. We have the notion of biequivalence of bicategories, the recognized "correct" notion of structural equality for bicategories; we write $\mathcal{A} \simeq \mathcal{B}$ for the biequivalence of bicategories $\mathcal{A}, \mathcal{B}$. We have particular finite one-way category $\mathbf{L}_{\text {bicat }}$ (having four levels, rather than three as in the previous two examples) so that a bicategory may be naturally considered as a $\mathbf{L}_{\text {bicat }}$-structure. For every bicategory $\mathcal{A}$, we have a particular anabicategory $\mathcal{A}^{\#}$, the saturation of $\mathcal{A} \cdot \mathcal{A}^{\#}$ is obtained by saturating the composition functors, and the identities (as functors from 1, the 
terminal category), and by other necessary adjustments. $\mathcal{A}^{\#}$ is also an $\mathbf{L}_{\text {bicat }}{ }^{-}$ structure. The basic fact it:

$$
\mathcal{A} \simeq \mathcal{B} \Leftrightarrow \mathcal{A}^{\#} \simeq \mathbf{L}_{\text {bicat }} \mathcal{B}^{\#}
$$

The verification of the facts $(2),(3),(4)$, though by no means difficult, consist in increasingly complex calculations. I believe that they are all special cases of a (future) general result on categorical structures of arbitrary dimension. In this, each structure in question is presented in the form of an $\mathbf{L}$-structure, for a specific one-way category $\mathbf{L}$. The categorical consideration provides one notion of equivalence, based on the morphisms, natural transformations, etc., in arbitrary dimensions connected to the original concept. The other notion of equivalence is that of $\mathbf{L}$ - equivalence. The would-be theorem is that the two coincide. Here, of course we mean that the two relations of equivalence coincide; the fuller notions, the two concepts of equivalence-carriers will have a more complex relation to each other.

I now have, for any fixed $n \in N$, a presentation of the Baez/Dolan $n$-categories as $\mathbf{L}_{n}$-structures for a particular (infinite) one-way category $\mathbf{L}_{n}$. Baez and Dolan themselves provided a notion of equivalence of $n$-categories. I am confident that it will turn out that the Baez/Dolan $n$-equivalence, and $\mathbf{L}_{n}$-equivalence coincide, although I still have to do the calculations for this.

Using $\mathbf{L}$-equivalence for $\mathbf{L}=\mathbf{L}_{n}$ and also for another, unspecified, FOLDS signature $\mathbf{L}$, I can give a precise formulation to the conjecture that the Baez-Dolan notion of $n$-category is "essentially finite", in the sense that by one can change the signature $\mathbf{L}_{n}$ to a finite FOLDS signature, with the concept of $n$ - category remaining the same "up to equivalence".

\section{Invariance under L-equivalence}

Let $\mathbf{L}$ be a one-way category, $M, N$, L-structures, $\mathcal{X}$ a context of variables and $\mathbf{a} \in M[\mathcal{X}], \mathbf{b} \in N[\mathcal{X}]$ valuations. We write $(M, \mathbf{a}) \simeq_{\mathbf{L}}(N, \mathbf{b})$ if there exist $(P, h, k): M \simeq_{\mathbf{L}} N$ and $\mathbf{x} \in P[\mathcal{X}]$ such that $\mathbf{x} \stackrel{h}{\mapsto} \mathbf{a}, \mathbf{x} \stackrel{k}{\mapsto} \mathbf{b}$. Each FOLDS-formula $\theta$ is preserved under $\mathbf{L}$ equivalence;

$$
\mathbf{a} \in M[\mathcal{X}: \theta], \quad(M, \mathbf{a}) \simeq_{\mathbf{L}}(N, \mathbf{b}) \Rightarrow \mathbf{b} \in N[\mathcal{X}: \theta] .
$$

$(\operatorname{Var}(\theta) \subset \mathcal{X})$. The proof of this is a straightforward verification, by induction on the complexity of $\theta$.

The generalization of this fact for intuitionistic logic, and for interpretations in a category suitable for intuitionistic logic ("Heyting category": see [MR2]) is, somewhat surprisingly, also true (the clause for $\exists$ is the natural one to show directly; in classical logic, the one for $\forall$ is a consequence since $\forall$ is expressible in terms of $\exists$; in the intuitionistic case, $\forall$ is not expressible in terms of $\exists$ ). 
We have a converse of this fact. Let $\varphi$ be a formula of FOL over $\mathbf{L}$ (see beginning of section 9 ); assume that the variables of $\varphi$ are among the ones in $\mathcal{X}$, a context of FOLDS over $\mathbf{L}$ (a variable of sort $X=K(\cdots)$ in FOLDS is understood, when in FOL over $\mathbf{L}$, to be a variable of sort $K$ ). Let $S$ be any theory in FOL over $\mathbf{L}$. Then, if $\varphi$ is invariant under L-equivalence for models of $S$ :

$$
M, N \vDash S, \quad M \vDash \varphi[\mathbf{a} / \mathcal{X}], \quad(M, \mathbf{a}) \simeq_{\mathbf{L}}(N, \mathbf{b}) \Rightarrow N \vDash \varphi[\mathbf{b} / \mathcal{X}],
$$

then there is a FOLDS formula $\theta, \operatorname{Var}(\theta) \subset \mathcal{X}$, such that $\varphi$ and $\theta$ are equivalent under $S$ :

$$
M \vDash S, \quad \mathbf{a} \in M I[\mathcal{X}] \Rightarrow M \vDash \varphi[\mathbf{a} / \mathcal{X}] \leftrightarrow M \vDash \theta[\mathbf{a} / \mathcal{X}] .
$$

This result is a special case of the Invariance Theorem stated below. Applied to $\mathbf{L}_{c a t}$, it together with the relation 10.(2), implies P.Freyd's theorem [F] characterizing first order formulas of category theory that are invariant under equivalence of categories.

To obtain similar results for more complex categorical structures, a stronger form of the Invariance Theorem is needed. To state it, we need the concept of an interpretation of a theory $T=(\mathbf{L}, \Sigma)$ in FOLDS in an ordinary first order theory $S$.

For the sake of definiteness, we restrict ourselves to classical FOLDS (the case of intuitionistic logic is also relevant). There is a "conceptual category" $[S]$ associated with $S$, made up of formulas of $S$. See [MR1]. An interpretation of $T$ in $S$ is a functor $\mathbf{L} \rightarrow[S]$, that is, on $[S]$-valued structure which is a model of $T$, one that makes all axioms in $\Sigma$ true.

There are two things lacking for this description to be an explicit definition. One is the $\mathbf{S}$-valued semantics, already mentioned at the end of section 8 ; the other is the definition of the particular $\mathbf{S}=[S]$.

Another way of giving the definition would proceed by associating an appropriate quantificational fibration (mentioned in section 9) with $T$, and one with $S$ too, and defining interpretation as the direct notion of structure-preserving morphism from one to the other.

However, the concept of an interpretation $I: T \rightarrow S$ of $T$ in $S$ is, we claim, intuitively clear. It is given by imitating the definition of "Set-valued model of $T$ ", and replacing in that definition sets and functions by $S$-definable sets and $S$-definable functions between the latter. Each object $K \in \mathbf{L}$ is mapped by $I$ to an abstract definable set $[\mathbf{x}: \varphi(\mathbf{x})]$ (read "the set of $\mathbf{x}$ such that $\varphi$ "), where $\varphi$ is a formula of $S$, and $\mathbf{x}$ is a finite tuple of variables containing all the free variables of $\varphi$. If $K$ is mapped to $[\mathbf{x}: \varphi(\mathbf{x})], K^{\prime}$ to $[\mathbf{y}: \psi(\mathbf{y})], \mathbf{x}$ and $\mathbf{y}$ are disjoint tuples, an arrow $f: K \rightarrow K^{\prime}$ is mapped to an abstract definable function $<\mathbf{x} \mapsto \mathbf{y}: \theta(\mathbf{x}, \mathbf{y})>$ (read " the function that maps $\mathbf{x}$ to $\mathbf{y}$ determined by the 
condition $\theta(\mathbf{x}, \mathbf{y})$ "); of course, it has to be provable in the theory $S$ that the said prescription is indeed a function defined on $[\mathbf{x}: \varphi(\mathbf{x})]$, with range contained in $[\mathbf{y}: \psi(\mathbf{y})]$. Further, the composition law of the category $\mathbf{L}$ has to be respected: every time $g f=h$ in $\mathbf{L}$, it has to be provable in $S$ that the "composite" of the definable functions corresponding to $f$ and $g$ is equal, in the sense of provability in $S$, to that corresponding to $h$. Finally, under the mapping described, formulas over $\mathbf{L}$ acquire meanings that are formulas over $L$; it is required that, in the sense of provability, the axioms of $T$ become true in $S$.

Given any interpretation $I: T \rightarrow S$ as described, there is associated, with any model $M$ of $S$, a model $M I$ of $T$. With $I$ construed as a functor $I: \mathbf{L} \rightarrow[S]$, and $M$ as a functor $[S] \rightarrow$ Set, $M I$ is the composite $M \circ I: \mathbf{L} \rightarrow$ Set.

The relevant example for the above concepts is the case of the FOLDS theory $T=T_{\text {sanafun }}=\left(\mathbf{L}_{\text {fun }}, \Sigma_{\text {sanafun }}\right)$ of (free-living) saturated anafunctors, and the ordinary theory $S=S_{\text {fun }}$, over the same $L=\mathbf{L}_{f u n}$, of all (free-living) functors. The construction $F \mapsto F^{\#}$ of the saturation of $F$, described in section 10 , is the same as the passage $F \mapsto F I$ for a suitable, and obvious, interpretation

$$
I: T_{\text {sanafun }} \rightarrow S_{f u n}
$$

For simplicity, we are going to talk about invariance of sentences (formulas without free variables) only. Note that an interpretation $I: T \rightarrow S$ maps any FOLDSsentence $\theta$ over $\mathbf{L}$ to a sentence $I(\theta)$ such that, for any $M \models S, M \vDash I(\theta)$ iff $M I \models \theta$. Thus, for instance, any sentence $\theta$ of FOLDS over $\mathbf{L}_{c a t}$ is translated by (1) into a sentence $\theta^{*}=I(\theta)$ such that, for any functo $F$,

$$
F \models \theta^{*} \Leftrightarrow F^{\#} \models \theta \text {. }
$$

Let us say that an $S$-sentence $\varphi$ is invariant under $\mathbf{L}$-equivalence (relative to $I$ ) if the implication

$$
M, N \vDash S, M \vDash \varphi, M I \simeq_{\mathbf{L}} N I \Rightarrow N \vDash \varphi
$$

holds universally.

Invariance Theorem Given an interpretation $T=(\mathbf{L}, \Sigma) \rightarrow S$ of a FOLDS theory in a FOL theory, an $S$-sentence $\varphi$ is invariant under $\mathbf{L}$ - equivalence if and only if it is expressible in FOLDS over $\mathbf{L}$ : there is a FOLDS sentence $\theta$ over $\mathbf{L}$ such that $S \vDash \varphi \leftrightarrow I(\theta)$.

The proof of the Invariance Theorem uses standard tools of model theory [CK].

As an application, we can, e.g., characterize the first-order sentences that may be asserted of a structure of the form $\mathbf{X} \stackrel{F}{\longrightarrow} \mathbf{A}$, consisting of two categories and a functor. Such a sentence is invariant under the equivalence explained in the last section iff it is equivalent, for structures of the given kind, to $\theta^{*}$, for 
some FOLDS-sentence $\theta$ over $\mathbf{L}_{f u n}$, where $\theta^{*}$ is the translate of $\theta$ for which $F \models \theta^{*} \Leftrightarrow F^{\#} \theta$.

Of course, there is a similar characterization theorem for sentences invariant under biequivalence of bicategories.

The full form of the Invariance Theorem refers to formulas with free variables rather than sentences.

The Invariance Theorem is, essentially, a preservation theorem in the sense of [CK], and as such, it can be construed as a purely syntactic result. Regarded purely syntactically, it then has a natural version for intuitionistic logic. The latter is easily translated into an equivalent semantical version, with reference to Kripke's semantics for intuitionistic logic. [M3] establishes the intuitionistic form the Invariance Theorem.

\section{References}

[BD1] J.C. Baez and J. Dolan, Higher dimensional algebra and topological quantum field theory. Journal of Mathematical Physics 36(1995), no. 11, 60736105 .

[BD2] J.C. Baez and J. Dolan, $n$-Categories - Sketch of a Definition. Letter to Ross Street, Nov. 29, 1995; corrected version as of Dec. 3, 1995.

[B] M. Barr, Respresentations of categories. J. Pure and Applied Algebra 41 (1986), 113-137.

[Ba] J. Barwise, Back and forth thru infinitary logic. In: Studies in Model Theory, ed. M.D. Morley. MAA Studies in Mathematics, Vol. 8. The Mathematical Association of America, 1973; pp. 5-34.

[Be] J. Benabou, Introduction to bicategories. In: Lecture Notes in Math., no. 47, Springer Verlag, 1967; pp. 1-77.

[Ben1] P. Benaceraff, What numbers could not be. Philosophical Review $\mathbf{7 4}$ (1965), 47-73.

[Ben2] P. Benaceraff, Mathematical truth. The Journal of Philosophy LXX, no. $19,1973,661-675$.

[B1] G. Blanc, Equivalence naturelle et formules logiques en theorie des categories. Archiv. math. Logik 19 (1978), 131-137.

[C] J. Cartmell, Generalised algebraic theories and contextual categories. Annals of Pure and Applied Logic 32 (1986), 209-243. 
[CK] C.C. Chang and H.J. Keisler, Model Theory. Third edition, North-Holland, 1990.

[Co] M. Coste, Localisation, spectra and sheaf representation. In: Applications of sheaves, Lecture Notes in Math., no. 753, Springer Verlag, 1979; pp. 212-238.

[D] R. Diaconescu, Axiom of choice and complementation. Proc. Amer. Math. Soc. 51 (1975), 176-178.

[F] P. Freyd, Properties invariant within equivalence types of categories. In: $\mathrm{Al}$ gebra, Topology and Category Theories, ed. A. Heller and M. Tierney, Academic Press, New York, 1976; pp. 55-61.

[FS] P.J. Freyd and A. Scedrov, Categories, Allegories. North-Holland, 1990.

GPS] R. Gordon, A.J. Power and R. Street, Coherence for tricategories. Memoirs Amer. Math. Soc. 117(1995), no. 558.

[Gr] A. Grothendieck, Expose VI, Revetements Etales et Groupe Fondamental; SGA1. Lecture Notes in Mathematics 224, Springer-Verlag, 1971; pp. 145-194.

[H] M. Hallett, Cantorian Set Theory and Limitation of Size. Oxford University Press, 1984.

[J] P.T. Johnstone, Topos Theory. Academic Press, 1977.

[K1] G.M. Kelly, Complete functors in homology I. Chain maps and endomorphisms. Proc. Cambridge Phil. Soc. 60 (1964), 721-735.

[K2] G.M. Kelly, Basic Concepts of Enriched Category Theory. London Math. Soc. Lecture Notes Series 64, Cambridge University Press, 1982.

[LS] J. Lambek and P. Scott, Introduction to Higher Order Categorical Logic. Cambridge University Press, 1986.

[ML] S. Mac Lane, Categories for the Working Mathematician. Springer-Verlag, 1971.

[MM] S. Mac Lane and I. Moerdijk, Sheaves in Geometry and Logic. A First Introduction to Topos Theory. 2nd corrected printing. Springer-Verlag, 1994.

[M1] M. Makkai, Generalized sketches as a framework for completeness theorems. To appear in three parts in J. Pure and Applied Algebra.

[M2] M. Makkai, Avoiding the axiom of choice in general category theory. $J$. Pure and Applied Algebra 108(1996), 109-173. 
[M3] M. Makkai, First-Order Logic With Dependent Sorts with Applications to Category Theory. Research monograph, to appear in the series Lecture Notes in Logic of Springer-Verlag.

[M4] M. Makkai, On the Baez/Dolan concept of $n$-category. In preparation.

[M5] M. Makkai, What are abstract objects? In preparation.

[M6] M. Makkai, An Algebraic Look at Propositional Logic. AILA Preprint no. 18 -iuglio/dicembre 1994. Associazione Italiana de Logica e sue Applicazioni.

[M7] M. Makkai, The fibrational formulation of intuitionistic predicate logic I: Completeness according to Godel, Kripke and Lauchli. Notre Dame Journal of Formal Logic 34 (1993), 334-377 (Part 1) and 471-498 (Part 2).

[MR1] M. Makkai and G.E. Reyes, First Order Categorical Logic. Lecture Notes in Mathematics, no 611, Springer-Verlag, 1977.

[MR2] M. Makkai and G.E. Reyes, Completeness theorems for intuitionistic and modal logic in a categorical setting. Annals of Pure and Applied Logic 72 (1995), 25-101.

[MP] M. Makkai and Robert Pare, Accessible Categories. Contemporary Mathematics, V. 104. American Mathematical Society, 1989.

[M-L] P. Martin-Löf, An Intuitionistic Theory of Types. In: Proc. Bristol Logic Colloquium, North-Holland, 1973.

[MPW] R. Milner, J. Parrow and D. Walker. A calculus of mobile process II. Information and Computation 100 (1) 41-77.

[S] R. Street, Fibrations in bicategories. Cah. de Top. et Geom. Diff. 21(1980), 111-160. 